\title{
Brain-wide quantification of the supraspinal connectome
}

Zimei Wang ${ }^{\mathrm{a}}$, Adam Romanski ${ }^{\mathrm{a}}$, Vatsal Mehra ${ }^{\mathrm{a}}$, Yunfang Wang ${ }^{\mathrm{b}}$, Benjamin C. Campbell ${ }^{\mathrm{c}}$, Gregory A. Petsko ${ }^{\mathrm{c}}$, Pantelis Tsoulfas ${ }^{\mathrm{b} *}$ and Murray Blackmore ${ }^{\mathrm{a} *}$

*Co-corresponding authors

${ }^{\mathrm{a}}$ Department of Biomedical Sciences, Marquette University, $53201{ }^{\mathrm{b}}$ Department of Neurological Surgery, Miami Project to Cure Paralysis, University of Miami Miller School of Medicine, Miami, FL 33136 'Helen and Robert Appel Alzheimer's Disease Research Institute, Weill Cornell Medicine, New York, NY 10021; Feil Family Brain and Mind Research Institute, Weill Cornell Medicine, New York, NY 10021

\section{murray.blackmore@marquette.edu}

ptsoulfa@med.miami.edu 


\section{Summary}

The supraspinal connectome is essential for normal behavior and homeostasis and consists of a wide range of sensory, motor, and autonomic projections from brain to spinal cord. Extensive work spanning a century has largely mapped the cell bodies of origin, yet their broad distribution and complex spatial relationships present significant challenges to the dissemination and application of this knowledge. Fields that study disruptions of supraspinal projections, for example spinal cord injury, have focused mostly on a handful of major populations that carry motor commands, with only limited consideration of dozens more that provide autonomic or crucial motor modulation. More comprehensive information is essential to understand the functional consequences of different injuries and to better evaluate the efficacy of treatments. Using viral retrograde labeling, 3D imaging, and registration to standard neuro-anatomical atlases we now provide a platform to profile the entire supraspinal connectome by rapidly visualizing and quantifying tens of thousands of supraspinal neurons, each assigned to more than 60 identified regions and nuclei throughout the brains of adult mice. We then use this tool to compare the lumbar versus cervically-projecting connectomes, to profile brain-wide the sensitivity of supraspinal populations to graded spinal injuries, and to correlate locomotor recovery with connectome measurements. To share these insights in an intuitive manner, we present an interactive web-based resource, which aims to spur progress by broadening understanding and analyses of essential but understudied supraspinal populations. 


\section{Introduction}

The brain's control of the body below the head is achieved largely by axonal inputs to spinal circuits, which then relay commands to the periphery through motor and autonomic output neurons. This supraspinal connectome is highly conserved in mammals, with multiple cell types distributed through the brainstem, midbrain, and motor cortex, each projecting axons to a subset of spinal levels and to selected cell types (Kuypers H. and Martin, 1982; Nudo and Masterton, 1988). A comprehensive and accessible approach for understanding supraspinal input is crucial for interpreting motor and autonomic behavior, and to treating conditions that disrupt descending signals such as stroke, disease, or injury to the spinal cord.

Extensive work spanning almost a century has employed orthograde degeneration, electrical stimulation, and axonal transport tracing methods to characterize the location and function of specific supraspinal neurons in various animals, providing a base of knowledge to understand supraspinal control (ten Donkelaar, 2000; Glees, 1946; Hoff and C H ff, 1932; Kuypers H. and Martin, 1982; Nudo and Masterton, 1988). Several efforts in rodents have provided more global information by performing retrograde tracing from selected spinal levels, followed by tissue sectioning and manual assignment of labeled cell bodies to regions within the brain (Lakke, 1997; Leong et al., 1984; Liang et al., 2011). Significant challenges, however, impede the distribution of this foundational knowledge and its application to the study of disease and injury-based disruptions. First, information about the location and types of supraspinal neurons is fragmented and not standardized across numerous studies (Tuszynski and Steward, 2012). Second, a high level of expertise is required to precisely identify brain regions from two-dimensional tissue series and to build a three-dimensional view of the connections (Economo et al., 2016; Gong et al., 2013; Oh et al., 2014). Third, tissue sectioning and imaging are laborious and time consuming. Thus, although several studies have been invaluable in mapping brain-wide patterns of descending input to the spinal cord, experiments to track dynamic changes after injury or disease remain impractical. Consequently, attention has remained focused on a relatively narrow set of supraspinal populations. For example, in the field of spinal cord injury, the vast majority of studies concern only a handful of descending populations, notably the corticospinal, rubrospinal, raphespinal, and broadly defined reticulospinal (Anderson, 2004; Fink and Cafferty, 2016; Kwon et al., 2002; Lu et al., 2012; Tuszynski and Steward, 2012; Xu et al., 1997). This attention is justified, as these 
regions serve important motor functions and comprise a majority of descending input (Lemon, 2008). On the other hand, dozens of additional brain regions also project to the spinal cord, many of which carry essential motor and autonomic commands (Liang et al., 2011). Without tools to easily monitor the totality of the supraspinal connectome, researchers lack even basic information regarding their sensitivity to injury, innate plasticity, or potentially disparate responses to potential pro-regenerative therapies.

Here we present a comprehensive and accessible approach to obtain detailed information about the number and location of descending projection neurons throughout the mouse brain. By combining retrograde viral labeling (Tervo et al., 2016), 3D imaging of optically cleared brains (Wang et al., 2018), and registration to standard neuro-anatomical space (Niedworok et al., 2016; Tyson et al., 2020), we rapidly identify the specific location of tens of thousands of supraspinal neurons. We present a web-based resource that compares the locations and quantity of supraspinal neurons that project to cervical versus lumbar levels. We further extend this approach to questions related to spinal cord injury by quantifying the region-specific sparing of distinct supraspinal populations in mice that received injuries of graded severity. Interestingly, this analysis revealed correlation between residual brain-spinal cord connectivity and locomotor function in a subset of neural populations. This approach provides an effective tool to disseminate detailed understanding of the supraspinal connectome, and a needed platform to rapidly achieve brain-wide profiling of disruption and restoration of brain-spinal cord connectivity after injury.

\section{Results}

Optimization of retrograde cell detection in cleared brain tissue

Prior work from our lab and others has combined retrograde viral labeling with 3D imaging to reveal supraspinal neurons and axons, with highly effective labeling in corticospinal tract (CST) and other neurons (Asboth et al., 2018; Frezel et al., 2020; Steward et al., 2021; Wang et al., 2018). Although informative, one limitation was dim label in some subcortical populations, compounded in the brainstem by optical interference from the nearby pyramidal and other descending tracts. We first considered whether replacing the prior fluorescent protein (FP) tdTomato with mScarlet (mSc), a bright red monomeric FP, might improve detection (Bindels et al., 2016). Adult mice received retrograde injection of AAV2-retro-mSc to lumbar spinal cord, followed two or four weeks later by 3DISCO-mediated clearing of brains and imaging by light-sheet fluorescence 
microscopy (LSFM) (Wang et al., 2018). Similar to prior results with tdTomato, CST neurons were labeled brightly (Figure 1A,C), but pontine and medullary populations were dimmer and partially obscured by the bright pyramidal tracts (Figure 1E,F). We therefore tested whether detection could be improved by localizing the retrograde FP to cell nuclei using histone $2 \mathrm{~B}$ (H2B) fused in frame. Indeed, two weeks after lumbar injection of AAV2-retro-H2B-mSc, the nuclearlocalized label greatly increased the number of discernable objects in the brainstem compared to cytoplasmic (Figure 1E-H). Spot detection in Imaris software confirmed an approximately twofold increase in the number of detected cells in the cortex, and more than 10-fold increase in detection in the brainstem $(249.6 \pm 56.3$ SEM vs. $3334.3 \pm 82.8$ SEM, Figure 1I-L). We conclude that nuclear-localized fluorescence greatly enhances cell detection in cleared tissue and utilized H2B constructs throughout this study.

To further optimize cell detection and allow flexibility in future labeling studies we tested mGreenLantern (mGL), a recently described green FP with enhanced brightness (Campbell et al., 2020). To directly compare the two different FPs, adult mice received lumbar (L1-L2) injection of mixed AAV2-retro-H2B-mGL and -mSc, followed two weeks later by brain clearing, 3D imaging, and nuclei detection using Imaris software. Based on location we classified retrogradely labeled nuclei into six groups: corticospinal, hypothalamic, red nucleus, dorsal pons, medullary reticular formation, and caudal dorsal medulla; note that additional nuclei existed outside these easily recognizable areas and are considered below (Supplemental Figure 1A-N). On average, both FPs labeled more than 15,000 cells across all regions. Compared to H2B-mSc, H2B-mGL yielded a significant elevation of counts in cortex, dorsal pons, and reticular formation ( $\mathrm{p}<.01,2$-Way ANOVA with post-hoc Sidak's) (Supplemental Figure 1-O). We compared counts in the right and left hemispheres and found statistically indistinguishable values, confirming that our spinal injection methods reached both sides equally (Supplemental Figure 2A). In addition, we compared cell counts at two versus four weeks post-injection and found no significant increase with time, indicating that detection is maximal by two weeks (Supplemental Figure 2B). Combined, these data establish an initial categorization and quantification of supraspinal brain regions in $3 \mathrm{D}$ space, reveal $\mathrm{H} 2 \mathrm{~B}-\mathrm{mGL}$ to be the most sensitive of the two FPs tested, and create consistent experimental parameters for the detection of supraspinal neurons. 

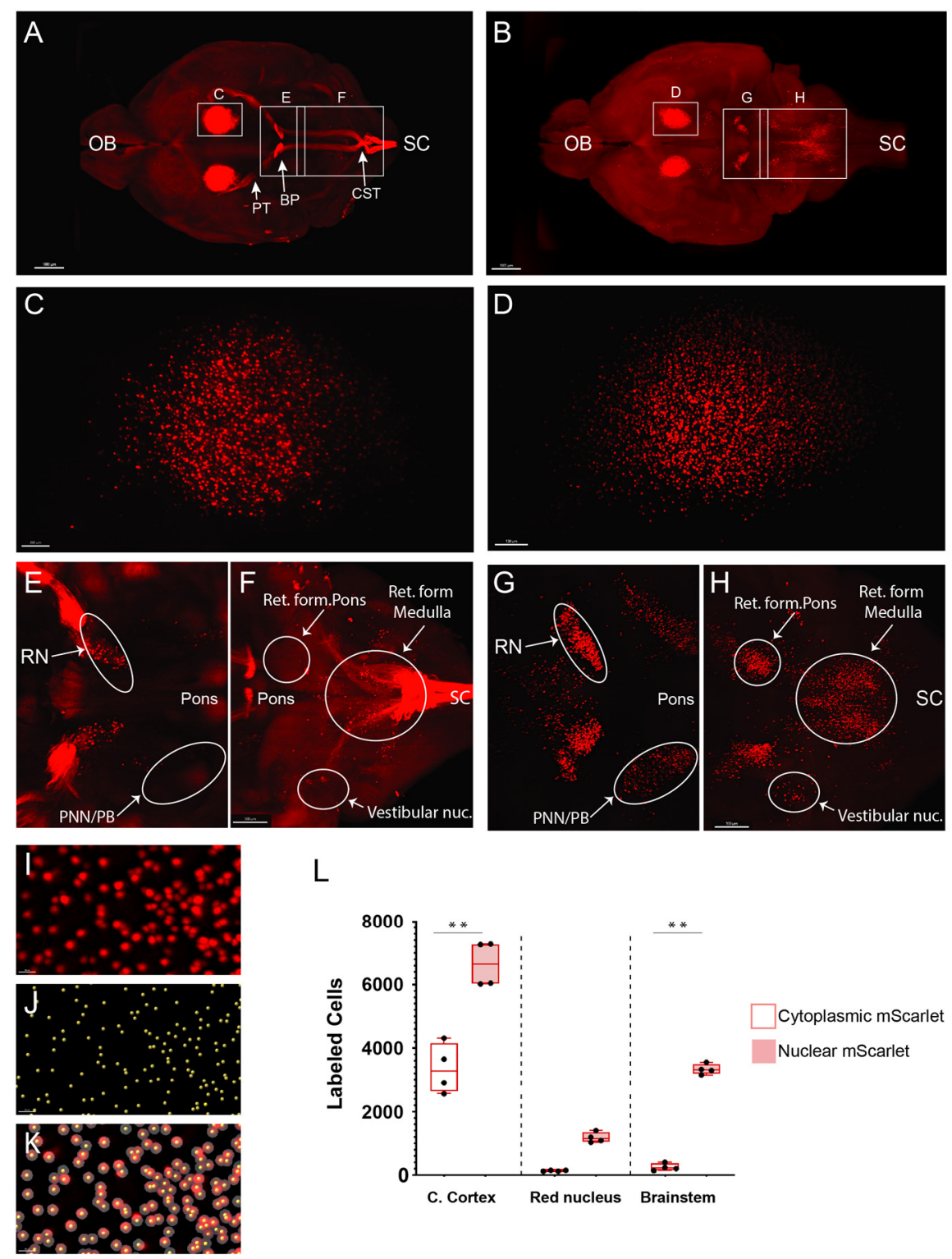

Figure 1: Nuclear localization of retrograde fluorophores enhances detection of supraspinal neurons in cleared brain tissue. Whole brains were cleared using 3DISCO and imaged by LSFM wo weeks after lumbar injection of AAV2-retro-mScarlet or H2BmScarlet. $(A, B)$ Dorsal views of whole brains with cytoplasmic $(A)$ and nuclear-localized (B) mScarlet. (C, D) High magnification views of cerebral cortex showing corticospinal tract neurons with cytoplasmic or nuclear label. $(E-H)$ Dorsal view of midbrain $(E, G)$ and brainstem $(F, H)$. Nuclear localized signal in $G, H$ shows no interference from axon tracts and increases the number of visible cells (arrows). (I) Higher magnification of cerebral cortex neurons labeled with nuclear localized mScarlet. (J) Yellow dots corresponding to the labeled nuclei in I. (K) Merged I and J images. L, Quantification of cells expressing the cytoplasmic and nuclear localized $\mathrm{mScarlet}$ in cerebral cortex, red nucleus and brainstem. Scale bar, A,B, $1000 \mu \mathrm{m}$; C,D, $200 \mu \mathrm{m}$; E-H, $500 \mu \mathrm{m}$. OB, Olfactory bulb; PT, Pyramidal tract; BP, basilar pons; SC, spinal cord; CST, cortico spinal tract; RN, Red nucleus; PNN/PB, Pedunculopontine and Parabrachial nuclei. ${ }^{* *} \mathrm{p}, .01,2$-Way Anova with post-hoc Sikak's, $N=$ 4 animals per group. 


\section{A pipeline for detection and spatial registration of supraspinal projection neurons}

The procedures outlined above greatly improve detection of supraspinal nuclei yet remain reliant on user-generated judgements regarding cell location. Manual registration, however, is likely only practical and accurate for large and isolated populations (e.g. corticospinal, rubrospinal, and others). An alternative solution is needed for brain regions with closely adjacent or intermingled populations (Tyson and Margrie, 2021). We therefore established an analysis pipeline using open-source tools to standardize brain registration and cell detection (Figure 2A-D). After tissue clearing and initial inspection in Imaris, image stacks were exported and pre-processed using ImageJ to create cell and background sets in standard orientation (Figure $\mathbf{2 A , B}$ ). Images were then registered and segmented using automated mouse atlas propagation (aMAP/brainreg), a wellvalidated tool to align 2D datasets with the 25um version of Allen Mouse Brain atlas (Kim et al., 2015; Niedworok et al., 2016; Tyson et al., 2020) (Figure 2C). We next used cellfinder, a deep learning model-based tool, for identification of labelled cells in whole brain images (Tyson et al., 2020) (Figure 2C). In conjunction with aMAP/brainreg, cellfinder assigns objects to 645 individual brain regions and quantifies the number in each. In addition, cellfinder produces detailed visualization of each optical slice, with defined brain regions outlined and labelled cells represented as overlaid spots (Figure 2C). For final visualization we used Brainrender (Claudi et al., 2021) which displays cellfinder output in an interactive 3D format registered a mouse brain atlas from the Allen Brain Institute (Figure 2D).

We applied this pipeline to approximately 40 animals that received spinal injections of varying location and graded spinal injuries. To facilitate understanding of the data beyond the descriptions below we also created a web-based interface (http://3dmousebrain.com/) that supplies quantification of supraspinal nuclei, static 2D visualization of defined supraspinal regions, and an interactive $3 \mathrm{D}$ representation that allows user-controlled rotation and zoom. Data from each experimental group are presented, with a focus on 69 brain regions that were found to contain supraspinal nuclei across the datasets (Supplemental Table 1 and Supplemental Figure 3). To standardize data presentation, each region was assigned an identification number in approximate caudal-to-rostral order, and with adjacent regions assigned adjacent numbers (Figure $2 \mathrm{C}$ and Supplemental Figure 3C). For each brain, the number of nuclei detected in each of these 69 supraspinal regions is provided as a graph on the web interface, and in Supplemental Table 1. 

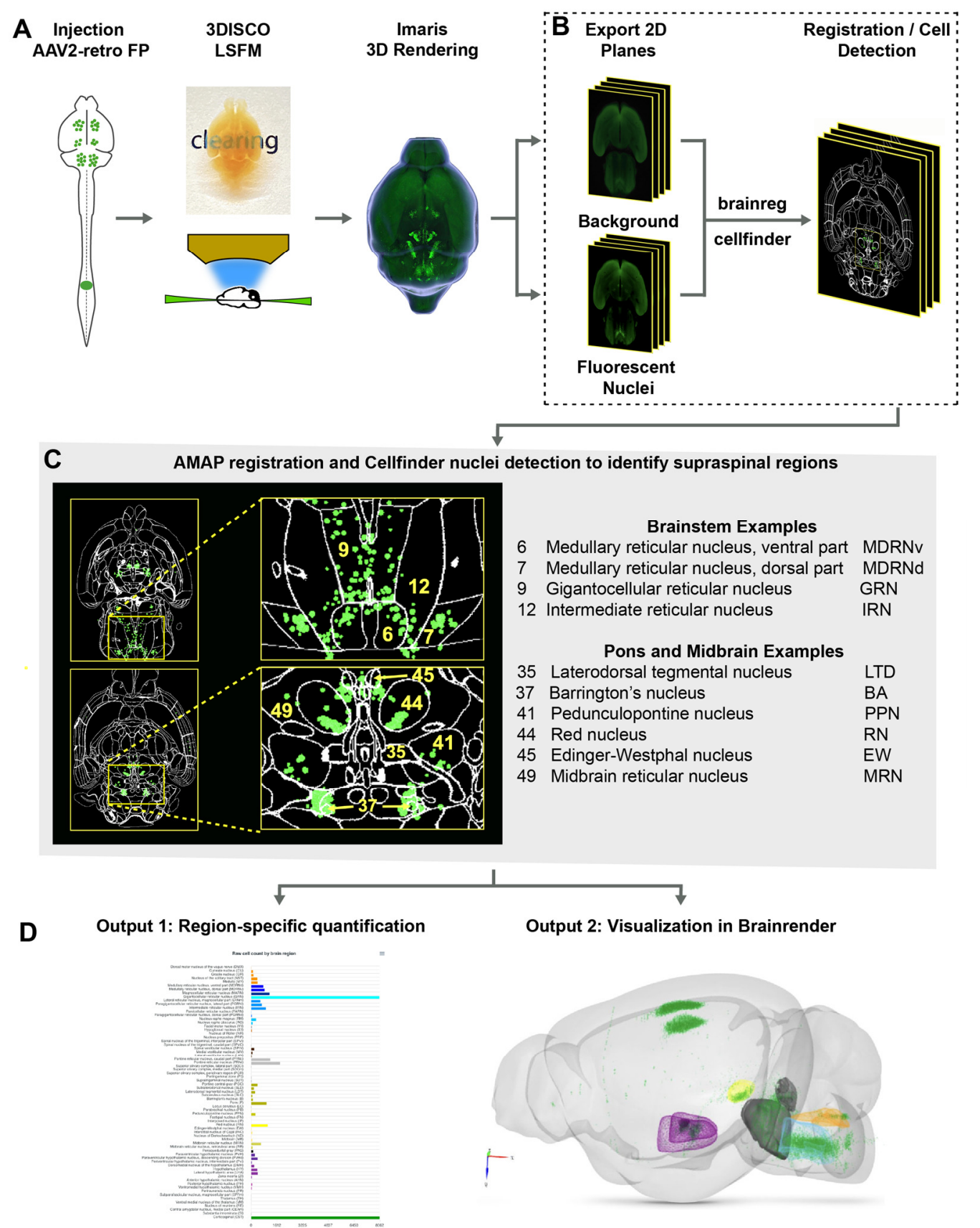

Figure 2: A pipeline and web resource for the detection and spatial registration of supraspinal projection neurons. (A) Tissue preparation and initial imaging. AAV2-retroH2B-mGL is injected to the spinal cord, followed by perfusion, tissue clearing, light-sheet imaging, and 3D processing using Imaris software. (B) Image registration. A complete series of background and fluorescent nuclei images are exported for registration to standard 3D space by brainreg and cell nuclei detection by cellfinder. (C) An example of cellfinder output, showing horizontal brain sections with brain regions outlined and detected cell nuclei indicated in green. (D) Example output available on "https://3Dmousebrain.com." On the left are quantitative nuclei counts for identified brain regions, and on the right is $3 \mathrm{D}$ visualization of supraspinal locations generated by Brainrender, an interactive python-based tool. 
We further simplified the output by presenting counts in 25 grouped categories, for example the spinal, medial, and lateral vestibular nuclei were grouped into a single vestibular category (Supplemental Figure 3D). Supplying the summarized readouts alongside the single-region data is intended to enhance accessibility to non-experts and to facilitate the detection of the differences among experimental groups outlined below. In summary, 3dmousbrain provides an accessible interface to share detailed brain-wide information about neurons that provide supraspinal input to the spinal cord.

\section{Brain clearing and registration quantifies supraspinal connectivity to the lower spinal cord}

We first applied the registration pipeline to examine connectivity from the brain to the lower spinal cord. Ten animals received injection of AAV2-retro-H2B-mGL to L1 spinal cord, followed two weeks later by perfusion, imaging, and analysis. On average, 31,219 nuclei were detected per brain (range 20,688 to 40,171). Complete nuclei counts are available in Supplemental Table 1. Below we highlight the variety of supraspinal populations identified by $3 \mathrm{D}$ registration, with reference to prior descriptions in rodent that help validate the automated findings. Note that we adopt nomenclature from the Allen Mouse Reference Atlas, which the cellfinder pipeline employs.

In the medulla the gigantocellular reticular formation (GRN) contained the largest mass of supraspinal neurons (Supplemental Figure 3A.8). The GRN is an evolutionarily conserved source of both pre-autonomic and motor axons, some of which synapse directly on spinal motor neurons (Aicher et al., 1995; Brownstone and Chopek, 2018; Hermann et al., 2003; Liang et al., 2016). More ventrally, labeled nuclei were present in the magnocellular reticular nucleus (MARN) (Supplemental Figure 3A.10). This region, alternatively referred to as the alpha and ventral region of the GRN, projects to the ventral horn and IML of the lower spinal cord. Notably, labeled nuclei also mapped to regions lateral to the GRN, including the Paragigantocellular Reticular Nucleus, lateral part (PGRNl) (Supplemental Figure 3A.10). This region contains spinally projecting neurons that initiate locomotion, as well as the ventral rostral medullary group that regulates blood pressure (Van Bockstaele et al., 1989; Capelli et al., 2017). A cluster of labeled nuclei was also located dorsally in the caudal medulla, in the vicinity of the solitary nucleus, consistent with prior work, and may be involved in linking visceral input to respiration and 
cardiovascular tone (Leong et al., 1984; Liang et al., 2011; Mtui et al., 1995) (Supplemental Figure 3A.3).

More rostrally in the brainstem, labeled nuclei were present in the spinal and medial vestibular nuclei, which project to spinal targets to mediate postural control (Supplemental Figure 3A.3). Labeled nuclei were also abundant in the pontine reticular nuclei, consistent with prior findings (Leong et al., 1984; Liang et al., 2011) (Supplemental Figure 3A.6). Although perhaps less well understood than medullary reticular populations, pontine reticular neurons have been linked to muscle atonia during sleep, startle responses, and to multi-segment postural adjustments during limb extension (Perreault and Giorgi, 2019; Takakusaki et al., 2016). More dorsally in the pons, labeled nuclei mapped to known supraspinal regions in and around the pontine central grey, including the locus coeruleus (LC), laterodorsal and sublaterodorsal tegmental nucleus (Cornwall et al., 1990; Leong et al., 1984; Liang et al., 2011; Peever and Fuller, 2016; Sluka and Westlund, 1992) (Supplemental Figure 3A.3). Labeled nuclei also registered to Barrington's nucleus (BAR), which plays a central role in the control of micturition and bowel control (Barrington, 1921; Verstegen et al., 2017) (Supplemental Figure 3A.2). Another prominent nucleus was the parabrachial nucleus (PBN), a sensory relay for inputs related to itch, pain, touch, and a range of autonomic controls including blood pressure and thermoregulation (Supplemental Figure 3A.3). (Chiang et al, 2019, Choi et al, 2020).

In the midbrain, supraspinal nuclei were prominently detected in the red nucleus (RN), as expected. Numerous nuclei also registered to midline regions including Edinger Westphal (EW) and the Interstitial Nucleus of Cajal (INC), known to supply supraspinal projections involved in postural adjustments and sympathetic functions including energy homeostasis (Supplemental Figure 3A.2) (Kozicz et al., 2011; Leong et al., 1984; Yu and Wang, 2020). Supraspinal cells were also detected in the midbrain reticular nucleus and pedunculopontine nucleus (PPN) (Supplemental Figure 3A.2), which lie within the mesencephalic locomotor region (MLR), a well-studied region in which stimulation triggers locomotion in a range of species including mice (Caggiano et al., 2018; Roseberry et al., 2016). Interestingly, although much MLR activity is known to act through reticular relays, the presence of direct supraspinal input from the PPN and MRN, noted here and elsewhere, indicate some role for direct spinal activation (Basbaum and Fields, 1979; Caggiano et al., 2018; Leong et al., 1984). 
Finally, in the forebrain, large clusters of nuclei were detected in the corticospinal tract region, as expected. These are considered in more detail below. Supraspinal neurons were also detected in the hypothalamus, where they separated into two prominent clusters, medial and lateral (Supplemental Figure 3A.7). The medial cluster mapped mostly to the paraventricular hypothalamic nucleus (PVH) and the adjacent descending paraventricular nucleus (PVHd). These are known to innervate autonomic circuitry in the lower spinal cord and to modulate functions including bladder control, sexual function, and blood pressure (de Groat et al., 2015; Holstege, 2005; Zhou et al., 2019). The lateral cluster spanned the dorsalmedial nucleus (DMH) and the lateral hypothalamic area (LHA). Although less well characterized than the PVH, prior work in the LHA has identified orexin-expressing neurons that project to all spinal levels with functions that include pain modulation (Van Den Pol, 1999; Swanson and Kuypers, 1980).

Overall, 3D imaging and registration located tens of thousands of neurons across the neuroaxis. Importantly, supraspinal neurons were mapped to distributed regions with broad correspondence to existing understanding of supraspinal connectivity. We also tested for variation in supraspinal numbers when injection sites were adjusted slightly to either lower lumbar (L4) or lower thoracic (T10). Interestingly, compared to L1 these cohorts showed no significant differences beyond a modest increase in CST and a modest decrease in GRN in T10-injected animals, highlighting the consistency of the approach (Supplemental Figure 4). We conclude that 3D imaging and neuro-anatomical registration provides a quantitative and global profile of neurons with spinal projections.

Use case 1: Brain-wide comparison of supra-lumbar versus supra-cervical connectomes.

Supraspinal populations can display topographic mapping with respect to innervation of spinal level. For example, motor cortex is divided loosely into forelimb and hindlimb regions, and the red nucleus contains lumbar-projecting neurons in more ventral/medial regions (Flumerfelt and Gwyn, 1974; Tennant et al., 2011). For many other supraspinal inputs, however, less is known regarding potential differences. We therefore performed $3 \mathrm{D}$ imaging and registration in animals that received both cervical (C4) injection of AAV2-retro-H2B-mSc and lumbar (L1) injection AAV-retro-H2B-mGL, enabling within-animal comparison (Figure 3A-D, H-J). Spatial registration in whole-brain data was not precise enough for definitive co-localization, and dual expression within single cells is considered in a separate analysis below (Figure 3E-G, K-M). 


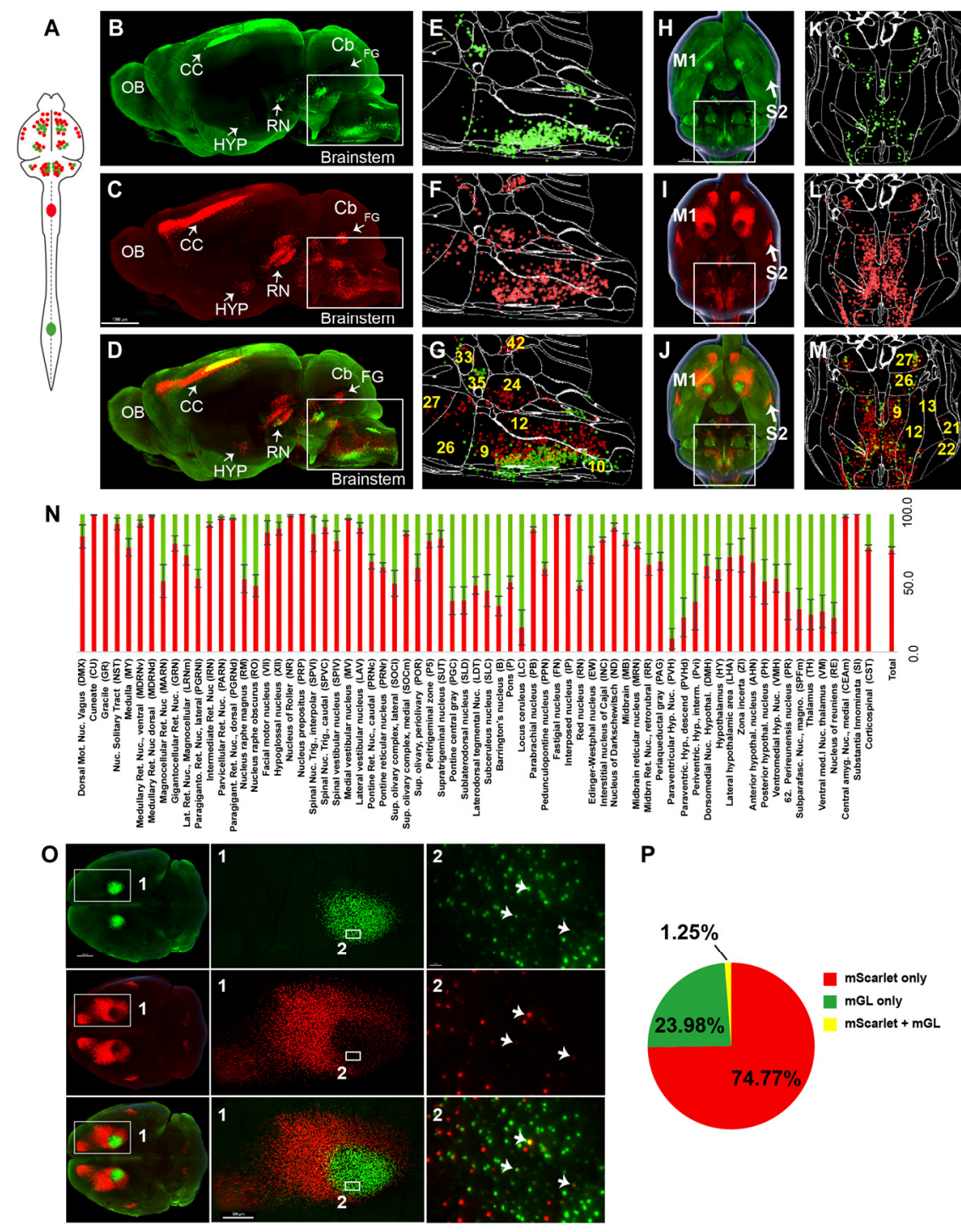

Figure 3: A brain-wide quantitative comparison of cervical and lumbar-projecting supraspinal neurons. (A) Experimental approach. AAV2-retro-H2B-mSc and $\mathrm{mGL}$ were delivered to C4 and L1 SC respectively, followed four weeks later by brain clearing, lightsheet microscopy, registration, and quantification. (B-G) Lateral view of brain and cellfinder output from brainstem regions. B,E show $\mathrm{mGL}$ (lumbar), C,F shows $\mathrm{mSc}$ (cervical) and $\mathrm{D}, \mathrm{G}$ show the overlay. Note the greater abundance of cervical signal in dorsal brainstem. $(\mathrm{H}-\mathrm{M})$ Dorsal brain views and cellfinder output of the same animals as B-G. H,K show mGL (lumbar), I,L show $\mathrm{mSc}$ (cervical), and J,M show the overlay. Note the relative abundance of cervical label in more lateral brainstem. (N) Quantification of the percent of cells in each brain region that project to cervical (red) or lumbar (green) spinal cord. (O) 3D dorsal views of cortical neurons labelled in green from L1 and red in C4. Inset 1 shows the entire M1 region, inset 2 shows intermingled cervical and lumbar region. Most cell nuclei are single-labeled; arrows show exceptions. (P) Quantification of the percent of neurons in M1 labeled with only mGL, only mSc, or dual-labeled. Scale bar, B,C,D, $1500 \mu \mathrm{m} ; \mathrm{H}, \mathrm{I}, \mathrm{J}, 1000 \mu \mathrm{m}$; O, Left pictures $1000 \mu \mathrm{m}$, middle $500 \mu \mathrm{m}$, right $30 \mu \mathrm{m} . \mathrm{n}=4$ biological replicates per group. OB, Olfactory bulb; CC, Cerebral Cortex; M1, Motor area 1; HYP, hypothalamus; RN, red nucleus; $\mathrm{Cb}$, Cerebellum; Fastigial nucleus; P, Pons; M. medulla. 
Here we focus on overall differences in cell counts between mSc (cervical) and mGL (lumbar) across brain regions. For each region we calculated the percent of nuclei that contained $\mathrm{mSc}$ signal, creating an index of cervical preference. Note that because cervical label was more abundant brainwide, comprising an average of $73 \%$ of all labeled nuclei (Figure 3N), we considered this level baseline and focused on deviation from $73 \%$ in each individual brain region.

Several patterns emerged. The medullary reticular formations, which occupy the most caudal region of the medulla, and the laterally positioned parvicellular nucleus both showed a predominant cervical projection, consistent their recently established involvements in forelimb control (Esposito et al., 2014; Ruder et al., 2021). In much of the brainstem a clear pattern was evident in which ventrally located populations projected to both lumbar and cervical regions, whereas more dorsally located populations were predominantly cervical (Figure 3B-G). The pontine reticular formations, however, showed a more balanced distribution. The nucleus prepositus and Roller nucleus, dorsally located in the medulla and involved in gaze tracking, showed mostly cervical projections (Figure 3N) (McCrea and Horn, 2006). The red nucleus was relatively balanced, but showed the topography in which ventral-medial neurons projected to lumbar cord (Figure 3B-D) (Flumerfelt and Gwyn, 1974; Liang et al., 2012; Wang et al., 2018). Neurons near the pontine central grey, including Barrington's nucleus, showed relative enrichment for lumbar labeling, consistent with known innervation of lumbar circuitry (Verstegen et al., 2017). Similarly, the paraventricular region of the hypothalamus was enriched for lumbar label, consistent with its known innervation of the IML cell column. Overall, these data provide comprehensive, brain-wide quantification of the relative distribution of cervical versus lumbar projections and provide $3 \mathrm{D}$ visualization of within-nuclei topography.

Next, focusing on the corticospinal tract, we used higher resolution imaging and spot detection in Imaris software to examine more closely the frequency of colocalized label from both cervical and lumbar cord. As expected, cervical mSc label was apparent in three discrete cortical areas: a large central mass (M1), a more rostrally located cluster (Rostral Forelimb Area, or RFA), and a lateral mass (S2) (Figure 30). The mGL label from lumbar cord was concentrated in a subregion of M1, centered medial and caudal to the main mass of cervically-labeled neurons. Interestingly, a rim of cervically labeled cells completely surrounded the lumbar region (Figure 30). Thus lumbar-projecting CST neurons can be described most accurately not as a separate 
population located caudally, but rather as nested within a broader region of cervical-projecting neurons (Steward et al., 2021). Interesting, the lumbar-projecting region also displayed sparse cervical label. Closer examination, however, revealed that these cervical-labeled cells were largely distinct from the surrounding lumbar-labeled cells, such that definitive co-localization was detected in only $1.25 \%$ of neurons (Figure 3P). These data suggest that although dual cervical/lumbar collateralization by single CST axons may occur in juvenile mice (Kamiyama et al., 2015), by adulthood collateralization is highly region-specific (Steward et al., 2021).

\section{Use case 2: Application to spinal injury}

We next applied whole-brain imaging and quantification to questions related to spinal cord injury. We first tested the ability of AAV2-retro to transduce neurons in the chronically injured spinal cord. Our prior work indicated AAV2-retro's efficacy in some cell types immediately after injury(Wang et al., 2018), but this conclusion was only qualitative and did not examine more extended and clinically relevant time points. We therefore injected AAV2-retro-H2B-mGL six weeks after a complete crush of thoracic spinal cord, followed two weeks later by tissue analyses (Figure 4A). Examination of the crush site in sections of spinal cord confirmed injury completeness, as evidenced by a lack of astrocytic bridges and lack of retrograde label distal to the injury (Figure 4B). In cleared brains, examination of retrograde mGL showed a broad distribution of signal (Figure 4C-G), and nuclei counts in registered brain regions did not differ significantly from those found previously in uninjured animals with similar thoracic injections (Figure 4H). These data quantitatively verify AAV2-retro's ability to effectively deliver transgenes to a wide diversity of cell types in the chronic phase of injury.

We next applied whole-brain quantification to a central challenge in SCI research, the issue of injury variability. In both the clinic and the laboratory, spinal injuries are often incomplete and leave inconsistent numbers of spared connections in each individual (Fouad et al., 2021). To generate a range of injury severities adult mice received mild, moderate, or severe crush injury to T10 spinal cord, using stoppers of defined thickness to control the width of compression (Cho et al., 2010). AAV2-retro-H2B-mGL was injected to L4 spinal cord seven weeks post-injury and tissue was analyzed two weeks post-injection. First, viral targeting and injury severity were assessed in spinal tissue sections (Figure 5A-F). Example images of 4 evenly spaced horizontal 

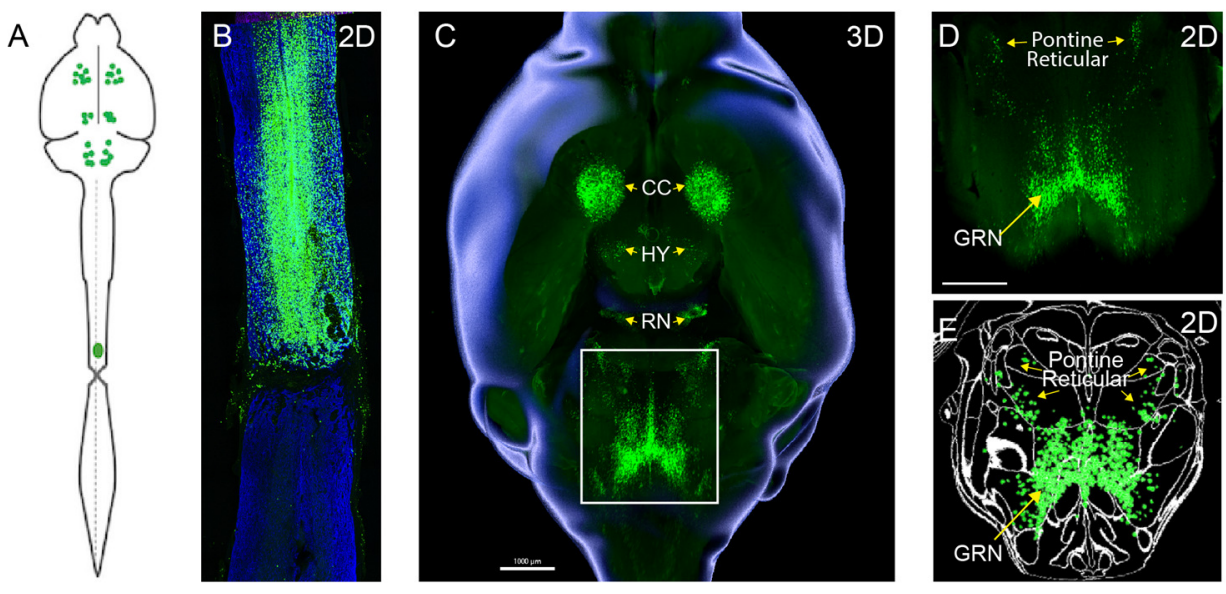

$\mathrm{F}$
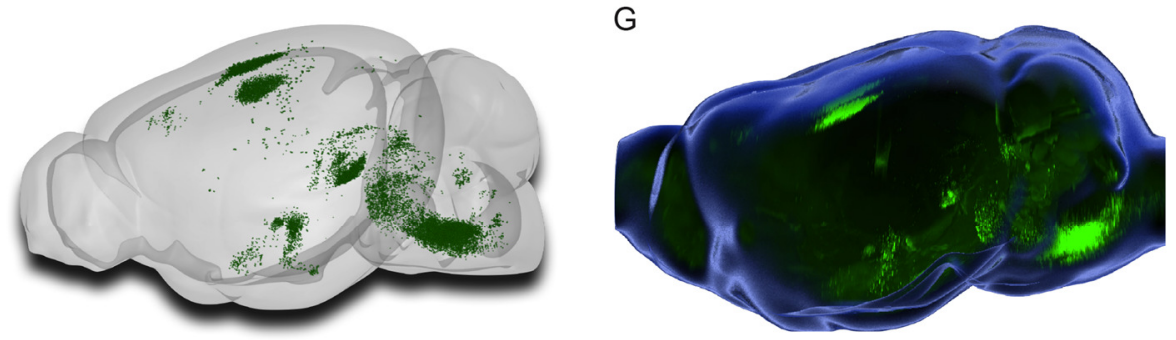

$\mathrm{H}$

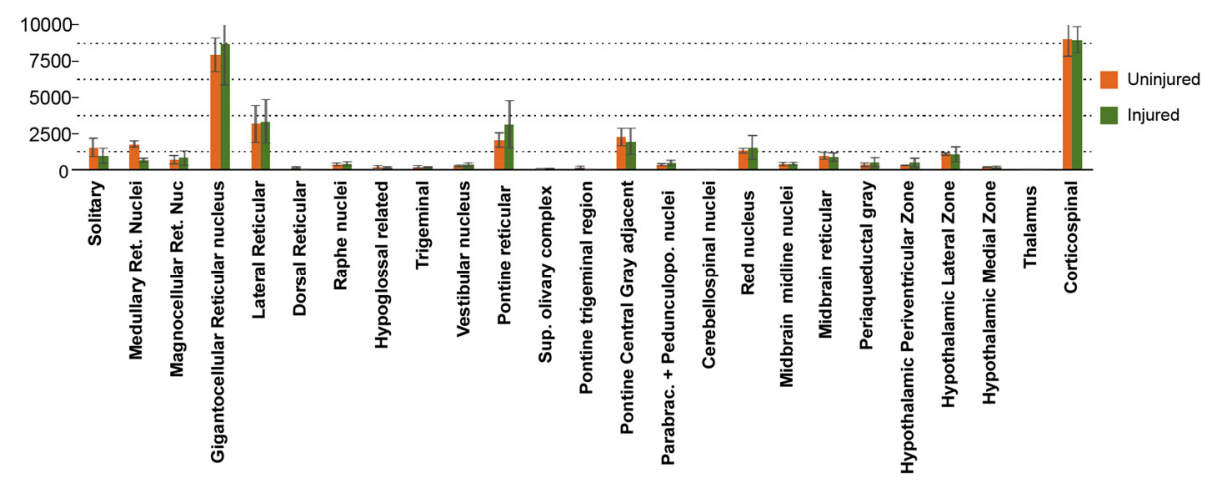

Figure 4: retro-AAV2 effectively transduces neurons when delivered to the chronically injured spinal cord. (A) Experimental design. A complete crush injury was delivered to lower thoracic spinal cord, followed six weeks later by injection of AAV2-retro-H2B-mGL rostral to the injury. Two weeks post-injection animals were euthanized, brains were cleared and imaged with light-sheet microscopy, and images were processed for registration and quantification by aMAP and cellfinder. (B) Horizontal spinal section stained for GFAP (blue), confirming the complete crush and verifying transduction (green) rostral but not caudal to the injury. (C-E) Ventral views of the brain from the same animal of section in $B$. C shows a 3D overview, $D$ shows a higher magnification view of the brainstem, and $E$ shows cellfinder output with retrograde $\mathrm{mGL}$ detection in green. (F) Brainrender output showing lateral views of the same brain in $C$. $(G)$ Imaris 3D generated equivalent lateral view of the brain in $F$. (H) Quantification of retrograde nuclei detected in 25 brain regions, comparing uninjured animals (orange) to chronically injured animals (green). No regions displayed statistical differences ( $p>.05,2-$ WAY ANOVA with post-hoc Sidak's). N = 4 animals per group. 
sections are provided in Supplemental Figure 5. As expected, mild injuries displayed elevated GFAP at the crush site but overall astrocytic continuity (Figure 5A, B). In contrast, severe injuries showed complete gaps in GFAP at the site of injury, with no detectable astrocytic bridges (Figure 5 E, F). Moderate injuries were highly variable and sometimes asymmetrical right to left (Figure 5C, D). Overall, as intended, the injuries displayed a wide range of severity.

To assess residual brain-spinal cord connectivity across the brain supraspinal neurons were registered and quantified by the pipeline described in Figure 3 (Figure 5G-L). Raw values for all brain regions are provided in Supplemental Table 1, and Figure 5 M-O shows values normalized to region counts in uninjured mice, thus creating an index of sparing for each region. Mice that received severe injuries showed a maximum of 29 labeled cells brain-wide, confirming disruption of descending axon tracts (Figure 50). In contrast, mild injuries averaged only a $43 \%$ reduction in retrograde label, with high variability between different supraspinal populations (Figure 5J,M). For example, the CST was strongly affected, averaging less than $20 \%$ sparing, while neurons near the pontine central grey and the red nucleus averaged $83.3 \%$ and $75.1 \%$ sparing, respectively (Figure 5M). The moderate injury group showed a brain-wide average of $24.2 \%$ sparing, also with high variability between animals (range $3.4 \%$ to 36.3\%) (Figure 5K,N). Similar to mildly injured animals, CST neurons were affected more strongly than other populations such as the PGC and $\mathrm{RN}$, although the animal with the highest overall sparing showed an unusual pattern of nearly $70 \%$ persistence of the corticospinal tract (Figure 5N). In summary these data confirm the ability of brain-wide analysis to detect overall sparing differences in groups of animals that received injuries of different severity, and more importantly to detail differences in the injuries' effects on individual animals and on individual cell populations.

Examination of spinal injuries showed cases in which tissue sparing was confined to one side of the midline. We therefore separated nuclei counts left and right and compared them to the spinal cord histology. Supplemental Figure 6A shows an example of a highly lateral injury and the corresponding brain imaging and quantification (Supplemental Figure 6B-D). Spared neurons showed asymmetry across the midline, consistent with the injury and the known pattern of ipsiversus contralateral innervation. For example, spared CST and RN neurons were almost completely contralateral to the injury, while spared neurons in the reticular and hypothalamic 


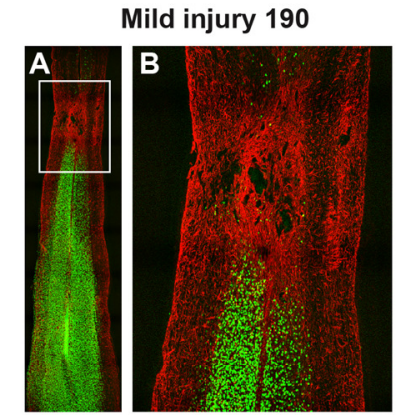

G
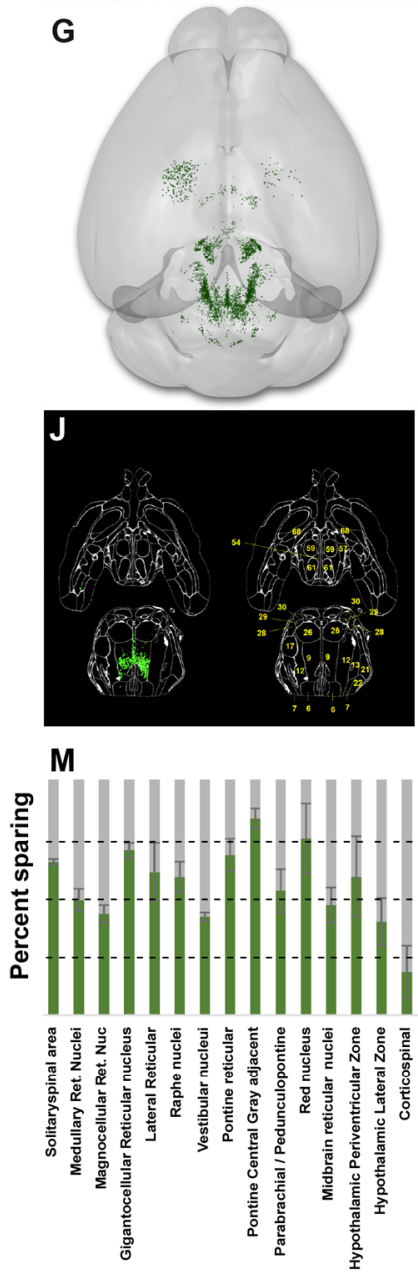

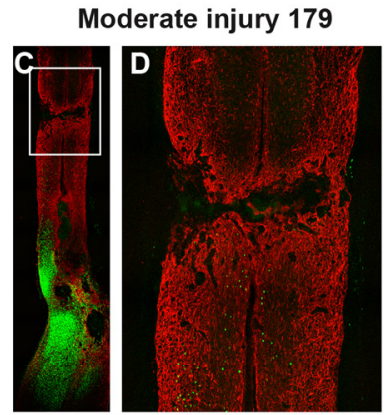

H
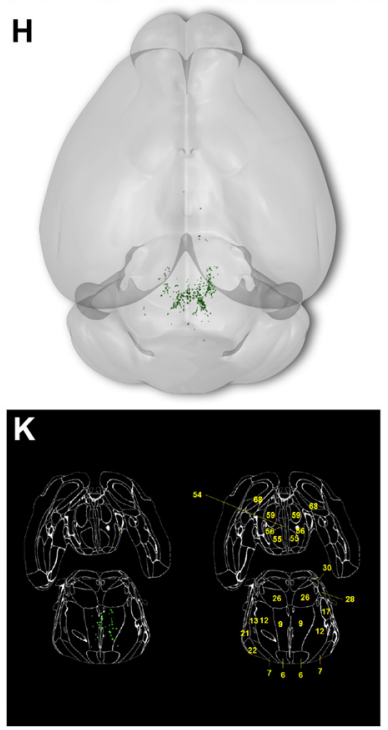

$\mathbf{N}$

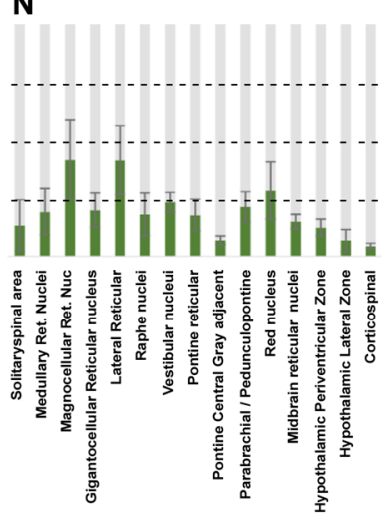

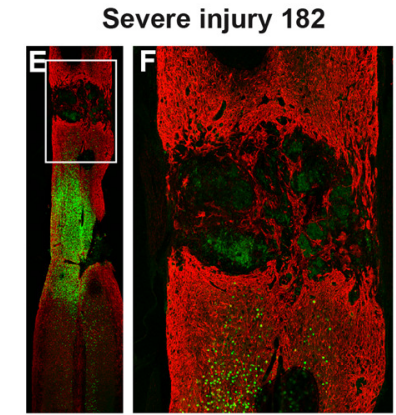
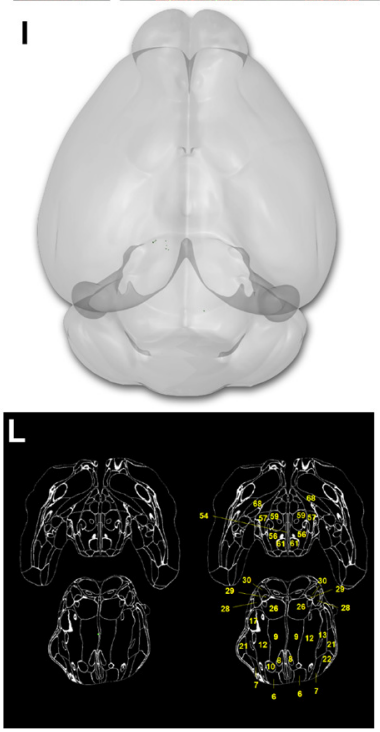

0

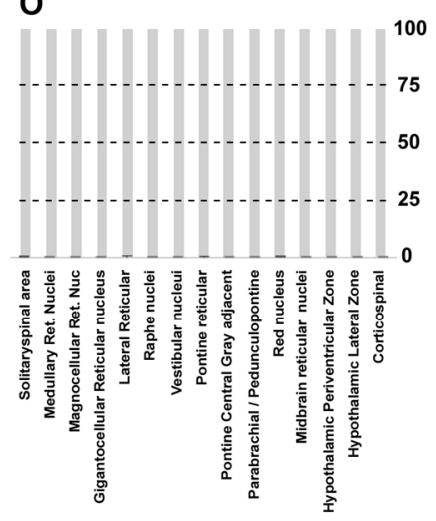

Figure 5: Brain clearing and registration quantifies region-specific sparing across a range of spinal injury severities. (A-F) Animals received thoracic crush injuries of controlled widths, followed eight weeks later by injection of AAV2-retro-H2B-mGL to L1 spinal cord. Horizontal spinal cord sections show mild $(A, B)$, moderate $(C, D)$, or severe $(E, F)$ injury, with GFAP in red and viral transduction in green. (G-L) Dorsal views of the brains from the same animals of the spinal cord sections above, showing progressive reduction in the number of retrogradely labeled neurons as injury severity increases. G-I show Brainrender depictions of whole brain, J-L show one 2D cellfinder plane output with brain regions outlined and detected $\mathrm{mGL}$ in green on the right panels. (M-O) Quantification of percent sparing in identified brain regions, with the average value from uninjured animals set as $100 . \quad N=3$ mild, 6 moderate, and 3 severely injured animals. 
nuclei were predominantly ipsilateral. Thus, when spinal injuries are variable across the midline, brain clearing and registration can quantify asymmetry in residual brain-spinal cord connectivity.

We next asked how indexes of sparing correlate with functional recovery from spinal injury as assessed by the BMS motor score, a well-established measurement of hindlimb function and interlimb coordination (Basso et al., 2006). As expected, BMS scores averaged lower in animals that received severe injury versus moderate or mild (Supplemental Figure 7A). Notably, however, within each group the scores varied widely. Combining all injury groups, we first examined the correlation between BMS scores and the size of the spinal injury, measured in spinal sections and defined as the GFAP-negative region bounded by gliosis. We identified a significant negative correlation between injury size and BMS score, but consistent with many prior findings in the field, injury size could explain only some of the variability in the data $\left(\mathrm{R}^{2}=0.53\right.$; Supplemental Figure 7B-E). We hypothesized that variability in motor recovery may also reflect inter-animal differences in the amount of sparing of supraspinal neurons in selected brain regions, and therefore created a correlation matrix to test for the potential relationship between locomotor scores and the number of spared neurons in each individual brain region (Figure 6A,B). Counts in some regions, such as the CST, hypothalamus, and lateral reticular formation correlated poorly with hindlimb function, and regression slopes did not differ significantly from zero $(\mathrm{R}<0.5, \mathrm{p}>.05$, simple linear regression). In contrast, in eleven brain regions, notably the gigantocellular reticular nucleus, the red nucleus, the pedunculopontine nucleus, and the pontine central grey, regression slopes differed significantly from zero with $\mathrm{R}$ values that varied between 0.75 and 0.93 at different timepoints. Interestingly, the number of spared propriospinal neurons in cervical spinal cord, counted in cleared tissue between $\mathrm{C} 2$ and C6, also correlated well with functional recovery, highlighting the potential importance of cervical neurons as a supralumbar control center $(\mathrm{R}=0.83$, slope $<0$ p=.0018, simple linear regression) (Supplemental Figure 8) (Zholudeva et al., 2021). Overall, these data support the utility of whole-brain imaging and quantification to partially explain variability in functional outcomes after spinal injury. 
A

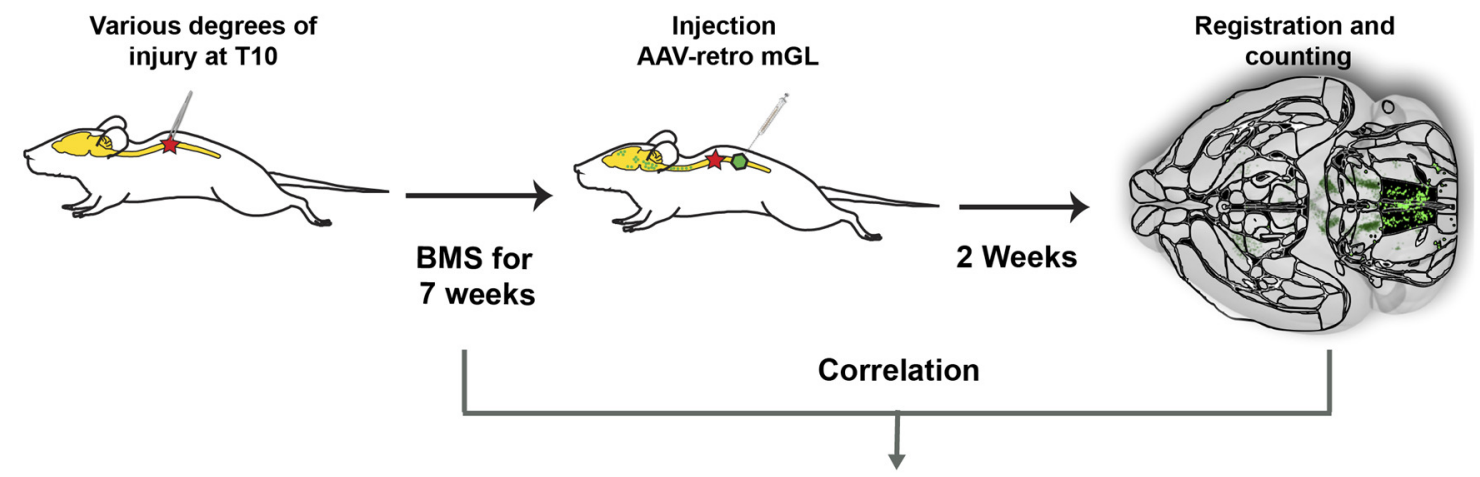

B

\begin{tabular}{|c|c|c|c|c|c|c|c|c|}
\hline & $\begin{array}{c}\text { Pre } \\
\text { Injury }\end{array}$ & 1 week & 2 weeks & 3 weeks & 4 weeks & 5 weeks & 6 weeks & 7 weeks \\
\hline Solitariospinal Area & -0.03 & 0.73 & 0.82 & 0.65 & 0.62 & \begin{tabular}{|l|} 
\\
\end{tabular} & 0.55 & 0.56 \\
\hline Medullary Reticular Nuclei & 0.04 & 0.66 & 0.64 & 0.54 & 0.57 & 0.50 & 0.49 & 0.50 \\
\hline Magnocellular Ret. Nuc. & 0.19 & 0.54 & 0.40 & 0.41 & 0.34 & 0.31 & 0.30 & 0.30 \\
\hline Gigantocell. Retic. Nuc. ${ }^{*}$ & -0.23 & 0.84 & 0.87 & 0.79 & 0.82 & 0.75 & 0.75 & 0.75 \\
\hline Lateral Reticular Nuclei & -0.35 & 0.46 & 0.68 & 0.46 & 0.59 & 0.53 & 0.52 & 0.53 \\
\hline Dorsal Reticular Nucleus & -0.34 & 0.43 & 0.64 & 0.44 & 0.56 & 0.52 & 0.51 & 0.52 \\
\hline Raphe Nuclei* & -0.26 & 0.80 & 0.67 & 0.76 & 0.71 & 0.66 & 0.65 & 0.65 \\
\hline Medullary Trigeminal Area & -0.36 & -0.01 & 0.05 & -0.01 & 0.07 & 0.04 & 0.03 & 0.03 \\
\hline Vestibular Nuclei* & 0.00 & 0.81 & 0.82 & 0.73 & 0.68 & 0.62 & 0.61 & 0.61 \\
\hline Pontine Reticular Nuclei* & -0.23 & 0.80 & 0.84 & 0.71 & 0.76 & 0.75 & 0.74 & 0.74 \\
\hline Superior Olivary Complex & -0.39 & 0.44 & 0.67 & 0.45 & 0.58 & 0.53 & 0.53 & 0.53 \\
\hline Pontine Trigeminal Area ${ }^{*}$ & -0.27 & 0.80 & 0.84 & 0.78 & 0.81 & 0.73 & 0.73 & 0.74 \\
\hline Pontine Central Gray Area* & -0.22 & 0.93 & 0.95 & 0.87 & 0.87 & 0.83 & 0.82 & 0.82 \\
\hline Parabrach. / Pedunculopont.* & -0.27 & 0.89 & 0.82 & 0.82 & 0.79 & 0.82 & 0.81 & 0.82 \\
\hline Red Nucleus* & -0.29 & 0.81 & 0.73 & 0.70 & 0.75 & 0.75 & 0.74 & 0.74 \\
\hline Midbrain Midline Nuclei* & -0.31 & 0.71 & 0.60 & 0.63 & 0.65 & 0.76 & 0.75 & 0.76 \\
\hline Midbrain Reticular Nuclei ${ }^{*}$ & -0.18 & 0.84 & 0.80 & 0.73 & 0.74 & 0.77 & 0.76 & 0.76 \\
\hline Periaqueductal Gray* & -0.20 & 0.82 & 0.85 & 0.72 & 0.76 & 0.77 & 0.76 & 0.77 \\
\hline Hypo. Periventricular Zone & -0.04 & 0.54 & 0.57 & 0.41 & 0.54 & 0.49 & 0.49 & 0.48 \\
\hline Hypothalamic Lateral Area & 0.06 & 0.71 & 0.58 & 0.56 & 0.58 & 0.58 & 0.57 & 0.58 \\
\hline Hypothalamic Medial Area & 0.20 & 0.66 & 0.46 & 0.49 & 0.48 & 0.43 & 0.42 & 0.42 \\
\hline Thalamus & -0.13 & 0.55 & 0.48 & 0.44 & 0.53 & 0.46 & 0.46 & 0.45 \\
\hline Corticospinal & -0.15 & 0.53 & 0.32 & 0.44 & 0.43 & 0.55 & 0.54 & 0.55 \\
\hline
\end{tabular}

Figure 6. Residual connectivity in specific brain regions correlates with motor recovery after spinal cord injury. (A) Experimental design. Adult mice received graded spinal cord injuries followed by weekly BMS testing. Seven weeks post-injury animals received lumbar injection of retro-AAV2-H2B-mGL, followed two weeks later by perfusion, 3D imaging of brains, registration, and region-specific quantification of spared neurons. (B) shows a correlation matrix of $R$ values derived from linear regression between the number of spared neurons in each of 25 brain regions and weekly BMS scores. * indicates eleven regions the regression slope differed significantly from zero $(p<.05$, simple linear regression). 


\section{Discussion}

We present a new experimental approach and an associated web-based resource that provides comprehensive quantification and visualization of neurons that project from the murine brain to specific levels of the spinal cord. Supraspinal projecting populations are numerous and diverse, yet prior publications, particularly in the spinal cord injury field, have focused disproportionately on specific sets of nuclei and a handful of major pathways. The new approach presented here is needed to spur progress in two ways. First, the visualization tools offer an educational resource for diverse researchers with an interest in brain-spinal cord connectivity. Making supraspinal populations easier to identify and their complex distribution more understandable can help lower conceptual barriers that currently restrict a broader consideration of pathways that are important but understudied. Second, optimized fluorescent proteins for retrograde labeling and a pipeline for registration and quantification make it practical for the first time to assess post-injury connectomes across the entire brain in numerous animals within an experimental study. This approach thus opens the door to comprehensive analyses of changes in supraspinal connectivity in response to disease and injury, and conversely to profile without bias the brain-wide efficacy of pro-regenerative therapeutics.

The use of genetically encoded FPs has revolutionized neural mapping (Chen et al., 2013; Oh et al., 2014; Rodriguez et al., 2017). In parallel, tissue clearing methods now provide unprecedented access for defining circuits of interest (Ueda et al., 2020). Harsh chemical treatments and imperfect clarity of cleared tissue, however, require FPs of exceptional brightness and stability. Here we deployed two newer-generation FPs. We found that mScarlet, a derivative of the red fluorescent protein, displayed similar intensity to the brightest FP, tdTomato, while utilizing a smaller open reading frame (Bindels et al., 2016). mGreenLantern, a recently described Clover mutant, also proved to be exceptionally bright and to resist bleaching by clearing solutions (Campbell et al., 2020). Importantly, we found nuclear localization to dramatically enhance detection of retrogradely transduced neurons, most notably in the brainstem, an important source of supraspinal control. This likely reflects the relative concentration of fluorescence in the nucleus, augmented by slower protein turnover and reduced interference from intervening axon tracts. Our direct comparison of cytoplasmic versus nuclear labeling indicates that previous studies using cytoplasmic FPs, including our own and a recent description of CST neurons in cleared brains, 
likely underestimated the number of retrogradely transduced neurons (Steward et al., 2021; Wang et al., 2018). Thus nuclear-localized $\mathrm{mSc}$ and $\mathrm{mGL}$ expand the tool kit for neuronal labeling in cleared tissue and allow flexibility in dual labeling experiments.

Once neurons are well labeled, the next challenge is to assign precise locations and numbers (Tyson and Margrie, 2021). Prior work has employed retrograde tracing and manual scoring of brain sections to meticulously catalogue supraspinal populations, and in most cases this foundational work should be considered definitive (Leong et al., 1984; Liang et al., 2011). Indeed, the broad concordance of these prior human-curated efforts with our automated registration approach provides essential validation. Although these efforts have been invaluable in defining supraspinal connections, the sheer number of populations and their complex spatial relationships present a significant challenge to non-experts, particularly when information is presented in 2D or described in text with reference to anatomical landmarks. We addressed this challenge by building a pipeline from pre-existing tools that assigns anatomical location and provides quantification (Tyson and Margrie, 2021). In addition, we provide a custom graphical user interface that represents cell position in 3D to help generate intuitive insights between the data and brain regions. By focusing on connectivity between the brain and spinal cord this new resource fills a gap in existing web based neuro-anatomical atlases, which focus mostly on intra-brain circuitry. Overall, this resource is intended as a point of entry for researchers into the complexity of supraspinal connections, from which to proceed to the detailed, foundational literature as needed.

More than its instructive function, the key advance of this approach is to enable for the first time a brain-wide assessment supraspinal connectivity that is both quantitative and practical. This addresses several critical needs, particularly in fields that focus on disruptions of supraspinal circuitry such as spinal cord injury. First, a central challenge in the SCI field is the so-called neuroanatomical-functional paradox, which refers to the fact that the size of lesions in the spinal cord are poorly predictive of functional outcomes (Fouad et al., 2021). This unpredictability of SCI outcomes is a major stumbling block that has likely contributed to challenges of reproducibility in the field (Steward et al., 2012). The paradox is driven by variability in a constellation of factors including cell loss, demyelination, plasticity, and compensatory movements. Another central contributor, however, may simply be incomplete anatomical information. It is reasonable to hypothesize that function after a spinal injury would depend on the 
amount of residual supraspinal connectivity, yet until now it has not been possible to quantify this key variable from most supraspinal populations. With the methods presented here we demonstrate comprehensive quantification of the variability between injury types and between animals. Importantly, we found lesion size per se to correlate only partially with functional recovery, whereas the number of spared neurons in selected supraspinal populations correlated more strongly. It is important to note that these observations remain correlational and are based on just one type of injury and a single functional readout. Nevertheless, they illustrate the potential for 3D imaging, registration, and quantification to help resolve variability in outcomes that currently challenge the field.

This resource can also fill a need that derives ultimately from the demands of individuals that suffer from supraspinal disruptions such as spinal cord injury (SCI). Besides the well-studied locomotor and fine motor deficits, SCI also affects pain sensation, bladder and bowel control, sexual function, basic postural control, cardiovascular tone, thermoregulation, and even metabolism (Anderson, 2004). The supraspinal populations that serve many of these functions are known, yet their response to injury and to attempted pro-regenerative strategies are largely uncharacterized (but see Adler et al. 2017 for an example of CNS-wide profiling of input to neural progenitor grafts in the spinal cord) (Adler et al., 2017). Thus, even treatments that advance to clinical trials can do so with very limited information on how or if they influence axon growth in tracts beyond the major motor pathways. In this context, a method to rapidly assess gains in connectivity in non-motor systems, and in populations that likely modulate the major motor pathways, is needed to address the concerns of individuals with SCI and to sharpen pre-clinical predictions.

Finally, important caveats to the approach should be considered. First, automated detection of nuclei likely remains imperfect and is impacted by the image quality of LSFM, notably stretching in the $\mathrm{Z}$ plane. Continued improvements with more isotropic acquisition in light sheet microscopy and in trained detection of nuclei will likely resolve these lingering issues (Chakraborty et al., 2019; Strack, 2021). A second caveat regards viral tropism, for example AAV2-retro appears less effective at transducing serotonergic cell types, thereby limiting assessment of raphe-spinal projections (Wang et al., 2018). This limitation will likely be addressed as additional retrograde variants are made (Davidsson et al., 2019). Finally, it is important to note 
that we have applied this approach only to descending inputs to the spinal cord, and not to ascending tracts. In principle a similar retrograde strategy could quantify neurons that give rise to ascending input, and a promising future direction would be to incorporate this information into predictive models for function after partial spinal injury. However, while these and other future developments are likely to further improve the approach, the present iteration provides information on an unprecedented scale and has yielded new insights into the complexity of supraspinal populations and their variable response to spinal injury.

\section{Acknowledgments}

This work was supported by a NIH/NINDS grant R01 NS083983 (M.B and P.T.) and the Bryon Riesch Paralysis Foundation (M.B.), The Miami Project to Cure Paralysis (P.T), and the Buoniconti fund (P.T). We would like to especially thank Troy Margrie, Adam Tyson, Luigi Petrucco and other investigators at the Brainglobe project for making available and helping to solve issues that arose during the installation and use of their neuroanatomical tools. We would like to thank Yania Ondaro-Martinez at the Miami Project Viral Core and Yan Shi at the Miami Project Image Core facility. We also thank Vance Lemmon for helpful comments and discussion of the manuscript.

\section{Author contributions}

P.T., and M.B. conceptualized and designed the study; Z.W., performed all the animal surgeries, viral injections, and immunohistochemical procedures; Y.W., P.T., and M.B. designed and did all the cloning of the various DNA constructs. Y.W., and P.T., cleared all the brains and image acquisition with LSFM. V.M. set up all the software tools from the Brainglobe and designed the web site. A.R. did all the analysis with Imaris. A.R., V.M., and M.B. did all the image modifications for use in cellfinder and brainrender. B.C., and G.P. designed and created the mGL. P.T. and M.B., did the analysis of all the data. M.B., wrote the first draft of the paper. B.C., P.T., and M.B., edited the manuscript. All authors contributed to the discussion of the experimental procedures, results and the manuscript.

\section{Declaration of interests}

All authors declare no competing interests.

\section{FIGURE LEGENDS}

Figure 1: Nuclear localization of retrograde fluorophores enhances detection of supraspinal neurons in cleared brain tissue. Whole brains were cleared using 3DISCO and imaged by LSFM wo weeks after lumbar injection of AAV2-retro-mScarlet or H2BmScarlet. (A,B) Dorsal views 
of whole brains with cytoplasmic (A) and nuclear-localized (B) mScarlet. (C, D) High magnification views of cerebral cortex showing corticospinal tract neurons with cytoplasmic or nuclear label. (E-H) Dorsal view of midbrain (E,G) and brainstem (F,H). Nuclear localized signal in G,H shows no interference from axon tracts and increases the number of visible cells (arrows). (I) Higher magnification of cerebral cortex neurons labeled with nuclear localized mScarlet. (J) Yellow dots corresponding to the labeled nuclei in I. (K) Merged I and J images. L, Quantification of cells expressing the cytoplasmic and nuclear localized mScarlet in cerebral cortex, red nucleus and brainstem. Scale bar, A,B, $1000 \mu \mathrm{m}$; C,D, $200 \mu \mathrm{m}$; E-H, $500 \mu \mathrm{m}$. OB, Olfactory bulb; PT, Pyramidal tract; BP, basilar pons; SC, spinal cord; CST, cortico spinal tract; RN, Red nucleus; PNN/PB, Pedunculopontine and Parabrachial nuclei.**p, .01, 2-Way Anova with post-hoc Sikak's, $\mathrm{N}=4$ animals per group.

Figure 2: A pipeline and web resource for the detection and spatial registration of supraspinal projection neurons. (A) Tissue preparation and initial imaging. AAV2-retro-H2BmGL is injected to the spinal cord, followed by perfusion, tissue clearing, light-sheet imaging, and 3D processing using Imaris software. (B) Image registration. A complete series of background and fluorescent nuclei images are exported for registration to standard 3D space by brainreg and cell nuclei detection by cellfinder. (C) An example of cellfinder output, showing horizontal brain sections with brain regions outlined and detected cell nuclei indicated in green. (D) Example output available on "https://3Dmousebrain.com." On the left are quantitative nuclei counts for identified brain regions, and on the right is $3 \mathrm{D}$ visualization of supraspinal locations generated by Brainrender, an interactive python-based tool.

\section{Figure 3: A brain-wide quantitative comparison of cervical and lumbar-projecting} supraspinal neurons. (A) Experimental approach. AAV2-retro-H2B-mSc and mGL were delivered to C4 and L1 SC respectively, followed four weeks later by brain clearing, light-sheet microscopy, registration, and quantification. (B-G) Lateral view of brain and cellfinder output from brainstem regions. B,E show mGL (lumbar), C,F shows $\mathrm{mSc}$ (cervical) and D,G show the overlay. Note the greater abundance of cervical signal in dorsal brainstem. (H-M) Dorsal brain views and cellfinder output of the same animals as B-G. H,K show mGL (lumbar), I,L show mSc (cervical), and J,M show the overlay. Note the relative abundance of cervical label in more lateral brainstem. (N) Quantification of the percent of cells in each brain region that project to cervical 
(red) or lumbar (green) spinal cord. (O) 3D dorsal views of cortical neurons labelled in green from L1 and red in C4. Inset 1 shows the entire M1 region, inset 2 shows intermingled cervical and lumbar region. Most cell nuclei are single-labeled; arrows show exceptions. (P) Quantification of the percent of neurons in M1 labeled with only mGL, only mSc, or dual-labeled. Scale bar, B,C,D, $1500 \mu \mathrm{m} ; \mathbf{H , I , J , ~} 1000 \mu \mathrm{m} ; \mathbf{O}$, Left pictures $1000 \mu \mathrm{m}$, middle $500 \mu \mathrm{m}$, right $30 \mu \mathrm{m} . \mathrm{n}=4$ biological replicates per group. OB, Olfactory bulb; CC, Cerebral Cortex; M1, Motor area 1; HYP, hypothalamus; RN, red nucleus; Cb, Cerebellum; Fastigial nucleus; P, Pons; M. medulla.

Figure 4: retro-AAV2 effectively transduces neurons when delivered to the chronically injured spinal cord. (A) Experimental design. A complete crush injury was delivered to lower thoracic spinal cord, followed six weeks later by injection of AAV2-retro-H2B-mGL rostral to the injury. Two weeks post-injection animals were euthanized, brains were cleared and imaged with light-sheet microscopy, and images were processed for registration and quantification by aMAP and cellfinder. (B) Horizontal spinal section stained for GFAP (blue), confirming the complete crush and verifying transduction (green) rostral but not caudal to the injury. (C-E) Ventral views of the brain from the same animal of section in B. C shows a 3D overview, D shows a higher magnification view of the brainstem, and E shows cellfinder output with retrograde mGL detection in green. (F) Brainrender output showing lateral views of the same brain in C. (G) Imaris 3D generated equivalent lateral view of the brain in F. (H) Quantification of retrograde nuclei detected in 25 brain regions, comparing uninjured animals (orange) to chronically injured animals (green). No regions displayed statistical differences ( $\mathrm{p}>$.05, 2-WAY ANOVA with post-hoc Sidak's). $\mathrm{N}=$ 4 animals per group.

\section{Figure 5: Brain clearing and registration quantifies region-specific sparing across a range of} spinal injury severities. (A-F) Animals received thoracic crush injuries of controlled widths, followed eight weeks later by injection of AAV2-retro-H2B-mGL to L1 spinal cord. Horizontal spinal cord sections show mild (A,B), moderate (C,D), or severe (E,F) injury, with GFAP in red and viral transduction in green. (G-L) Dorsal views of the brains from the same animals of the spinal cord sections above, showing progressive reduction in the number of retrogradely labeled neurons as injury severity increases. G-I show Brainrender depictions of whole brain, J-L show one $2 \mathrm{D}$ cellfinder plane output with brain regions outlined and detected $\mathrm{mGL}$ in green on the right 
panels. (M-O) Quantification of percent sparing in identified brain regions, with the average value from uninjured animals set as 100. $\mathrm{N}=3$ mild, 6 moderate, and 3 severely injured animals.

Figure 6. Residual connectivity in specific brain regions correlates with motor recovery after spinal cord injury. (A) Experimental design. Adult mice received graded spinal cord injuries followed by weekly BMS testing. Seven weeks post-injury animals received lumbar injection of retro-AAV2-H2B-mGL, followed two weeks later by perfusion, 3D imaging of brains, registration, and region-specific quantification of spared neurons. (B) shows a correlation matrix of $\mathrm{R}$ values derived from linear regression between the number of spared neurons in each of 25 brain regions and weekly BMS scores. * indicates eleven regions the regression slope differed significantly from zero $(\mathrm{p}<.05$, simple linear regression).

\section{METHODS}

\section{Resource availability}

Further information and requests for resources and reagents should be directed to and will be fulfilled by the Lead Contact, Murray Blackmore (murray.blackmore@marquette.edu). Certain materials have to be shared with research organizations for research and educational purposes under an MTA to be discussed in good faith with the recipient.

\section{Material availability}

Plasmids for the viral vectors are available from Addgene

\section{Data availability}

The datasets that support the findings of this study are available from the lead contact upon reasonable request. 


\section{Experimental model}

All animal procedures were approved by the Marquette University Institutional Animal Care and Use Committee and complied with the National Institutes of Health Guide for the Care and Use of Laboratory Animals. Adult female C57BL/6 mice (6-8 weeks old, 20-22 g) were used for these experiments. Age at day of surgery was eight weeks and mean weight was $20 \mathrm{~g}$. Group for initial fluorophore optimization were: mScarlet cytoplasmic, L1 injected, 4 weeks: 4 animals; H2BmScarlet, L1 injected, 4 weeks: 4 animals; H2B-mScarlet, L1 injected, 2 weeks: 7 animals; H2BmGreenLantern, L1 injected, 2 weeks: 7 animals. Group sizes for cleared and registered brains were: L1 injected: 10 animals; L3/4 injected: 5 animals; T10 injected: 4 animals; Cervical/lumbar co-injected: 4 animals; chronically injured: 3 animals; moderately injured: 6 animals; mildly injured: 3 animals; severely injured: 3 animals. The room temperature was set at $22^{\circ} \mathrm{C}\left( \pm 2^{\circ} \mathrm{C}\right)$ and room humidity was set at 55\% ( $\pm 10 \%)$. Mice were kept in a 12-h light/dark cycle with access to food and water ad libitum. Mice were checked daily by animal caretakers.

\section{Method Details}

Plasmid construction and cloning. We used 2 monomeric bright fluorescent proteins (FP) of similar size that encode for mGreenLantern (Campbell et al., 2020) and mScarlet (Bindels et al., 2016) and fused in frame with the core histone $\mathrm{H} 2 \mathrm{~B}$ in the amino terminus for nuclear localization of the FPs. Both fusions were synthetically constructed (Genscript, USA). The AAV mGreenLantern was constructed first by generating a synthetic cDNA optimized to the Human codon usage. The rat gene H2B/Histone H2B type 1- C/E/G (accession \#NP_001100822) was fused in frame to mGreenLantern with a linker of 8 amino acids (PPAGSPPA) between H2B and mGreenLantern. The fusion protein was cloned into pAAV-CAG-GFP (Addgene \#37825) by substituting the GFP with H2B-mGreenLantern using restriction enzymes BamHI and XhoI. For the mScarlet the human H2B clustered histone 11 (H2BC11) (accession \#NM_021058) was fused in frame without a linker and cloned into the pAAV-CAG-tdTomato (Addgene \#59462) using the sites KpnI and EcoRI at the 5 and 3 prime end respectively. Cytoplasmic mScarlet was cloned identically but with the H2B sequence omitted. rAAV2-retro-H2B-mGreenLantern was produced at the University of Miami viral core facility at the Miami Project to Cure Paralysis, titer = $1.4 \times 1013$ particles/ml. Virus was concentrated and resuspended in sterile HBSS and used without further dilution. The rAAV2-retro-mScarlet and rAAV2-retro-H2B-mScarlet was made by the 
University of North Carolina Viral Vector Core, titer $=4.3 \mathrm{X} 10^{\wedge} 12$ and $8.7 \times 1012$ particles $/ \mathrm{ml}$, respectively.

Spinal cord surgery. One microliter of rAAV2- retro particles was injected into the spinal cord with a Hamilton syringe driven by a Stoelting QSI pump (catalog \#53311) and guided by a micromanipulator (pumping rate: $0.04 \mu \mathrm{L} / \mathrm{min}$ ). AAV viral particles were injected at C4-C5, T10, L1, L4 vertebrae, unilateral injections at the same location, $0.35 \mathrm{~mm}$ lateral to the midline, and to depths of 0.6 and $0.8 \mathrm{~mm}$. Spinal cord crush injury. Adult female C57BL/6 mice (6-8 weeks old, 18-22 g) were anesthetized by ketamine/ xylazine. A laminectomy of vertebra T10-12 was performed using a fine pair of forceps and care was taken not to damage the dura. For each crush mouse, one of the two pairs of forceps were used to laterally compress the spinal cord to the corresponding thickness $(0.15$ and $0.4 \mathrm{~mm}$, depending on the pair of forceps $)$ and thereby establishing two groups with varying injury severity: the $0.15 \mathrm{~mm}$ and $0.4 \mathrm{~mm}$ injury groups. Carefully compress the spinal cord with forceps until the spacers connect. Hold in place for $15 \mathrm{sec}$ with 2 times to avoiding bias. A mild injury control group received identical treatment, including exposure, laminectomy, and placement of the forceps around the spinal cord, but no crush injury was performed

Tissue clearing and imaging. After 2-4 week expression, animals were euthanized with approved procedures. Animals underwent transcardial perfusion with $0.9 \%$ saline and $4 \%$ paraformaldehyde solutions in 1×-PBS (15710, Electron Microscopy Sciences). Whole brains and spinal cords were dissected and fixed overnight in $4 \%$ paraformaldehyde at $4{ }^{\circ} \mathrm{C}$ and washed three times in $\mathrm{PBS} \mathrm{pH}$ 7.4 , followed by storage in PBS. The dura was carefully and completely removed as residual dura can trap bubbles that prevent effective light-sheet microscopy. The brains and spinal cords were cleared using a modified version of the 3DISCO (Soderblom et al., 2015; Wang et al., 2018). Samples were incubated on a shaker at room temperature in 50, 80, and twice with $100 \%$ peroxidefree tetrahydrofuran (THF; Sigma-Aldrich, 401757) for $12 \mathrm{hr}$ each for a total of 2 days. Peroxides were removed from THF by using a chromatography column filled with basic activated aluminum oxide (Sigma- Aldrich, 199443) as previously described (Becker et al., 2012). The third day, samples were transferred to BABB solution (1:2 ratio of benzyl alcohol, Sigma-Aldrich, 305197; and benzyl benzoate, Sigma-Aldrich, B6630) for at least $3 \mathrm{hr}$. After clearing, samples were imaged the same day using light-sheet microscopy (Ultramicroscope, LaVision BioTec). The 
ultramicroscope uses a fluorescence macro zoom microscope (Olympus MVX10) with a 2× Plan Apochromatic zoom objective (NA 0.50). Image analysis and 3D reconstructions were performed using Imaris v9.5 software (Bitplane, Oxford Instruments) after removing autofluorescence using the Imaris Background Subtraction function with the default filter width so that only broad intensity variations were eliminated.

Imaris reconstructions. Image analysis and 3D reconstructions were performed using Imaris v9.5 software (Bitplane, Oxford Instruments) after removing autofluoresence using the Imaris Background Subtraction function with the default filter width so that only broad intensity variations were eliminated. Additionally, the entire brain was defined as an ROI in order to mask all background fluorescence outside the spinal cord surface. Artifact and nonspecific fluorescence surrounding the brain was segmented and removed using the automatic isosurface creation wizard based upon absolute intensity. Voxels contained within the created surface were set to zero and the remaining mask was used for all further analysis. Automatic segmentation of nuclei within specified ROIs was applied using the spots detection function and later superimposed on a maximum intensity projection volume rendering of the tissue. For some of the figures surfaces were created around the brains and spinal cords to make them more evident in the 3D reconstructions. Quality thresholds were set based upon visual inspection of the mixed model rendering for both spots and surfaces.

Immunohistochemistry. Adult animals were perfused with $4 \%$ paraformaldehyde (PFA) in $1 \times$ phosphate-buffered saline (PBS) (15710-Electron Microscopy Sciences, Hatfield, PA), brains, and spinal cords removed, and post-fixed overnight in 4\% PFA. Transverse sections of the spinal cord or cortex were embedded in 12\% gelatin in 1× PBS (G2500-Sigma Aldrich, St.Louis, MO) and cut via Vibratome to yield $100 \mu \mathrm{m}$ sections. Sections were incubated overnight with primary antibodies GFAP (DAKO, Z0334 1 : 500, RRID:AB_10013482), and rinsed and then incubated for $2 \mathrm{~h}$ with appropriate Alexa Fluor-conjugated secondary antibodies (R37117, Thermofisher, Waltham, MA, 1 : 500). Fluorescent images were acquired using Olympus IX81 or Zeiss 880LSM microscopes.

Analysis using computational neuroanatomy. We used the BrainGlobe's Initiative software (https://brainglobe.info) of interoperable Python-based tools for the analysis and visualization the 
data. For each brain we captured approximately 500 images. The captured was form ventral to dorsal to match the brainglobe default orientations (https://github.com/brainglobe). The 2D images first were assembled in Imaris v9.3.5 and 9.5 (Bitplane, Oxford Instruments). Two channels were created one to subtract the positive cell signal and generate another set with only background fluorescence. Images were exported to Image J to create a new set of TIFF files. The TIFF files of the sample images were further analyzed with a set of neuroanatomical computational tools developed for analysis of brain serial section imaging using light-sheet microscopy. First we fed, both set of images, background and positive signal images into the cellfinder a deep-learning network (Residual neural Network) to detect the positive cells (https://github.com/brainglobe/cellfinder) followed by registration and segmentation into a template brain with anatomical annotations based of the Allen Reference Mouse Brain Atlas (https://github.com/brainglobe/brainreg) and finally visualized with the brainrender (https://github.com/brainglobe/brainrender).

Quantification and Statistics. Throughout the manuscript means are used as summary values and standard error of the mean (SEM) as the indicator of variability. Data were tested for assumptions of parametric tests using Kolmogorov-Smirnov and Levene's tests. Values for N, the specific tests and post-hoc analyses, and p values are provided in figure legends and in the test of the Results section. All manual quantification, including behavioral assessment and measurements of lesion size, were performed by blinded observers. Statistical analyses were performed using Prism (Graphpad).

\section{References}

Adler, A.F., Lee-Kubli, C., Kumamaru, H., Kadoya, K., and Tuszynski, M.H. (2017). Comprehensive Monosynaptic Rabies Virus Mapping of Host Connectivity with Neural Progenitor Grafts after Spinal Cord Injury. Stem Cell Reports 8, 1525-1533.

Aicher, S.A., Reis, D.J., Nicolae, R., and Milner, T.A. (1995). Monosynaptic projections from the medullary gigantocellular reticular formation to sympathetic preganglionic neurons in the thoracic spinal cord. J. Comp. Neurol. 363, 563-580.

Anderson, K.D. (2004). Targeting recovery: priorities of the spinal cord-injured population. J. Neurotrauma 21, 1371-1383.

Asboth, L., Friedli, L., Beauparlant, J., Martinez-Gonzalez, C., Anil, S., Rey, E., Baud, L., Pidpruzhnykova, G., Anderson, M.A., Shkorbatova, P., et al. (2018). Cortico-reticulo-spinal 
circuit reorganization enables functional recovery after severe spinal cord contusion. Nat. Neurosci. 21, 576-588.

Barrington, F.J.F. (1921). The relation of the hind-brain to micturition. Brain 44, 23-53.

Basbaum, A.I., and Fields, H.L. (1979). The origin of descending pathways in the dorsolateral funiculus of the spinal cord of the cat and rat: Further studies on the anatomy of pain modulation. J. Comp. Neurol. 187, 513-531.

Basso, D.M., Fisher, L.C., Anderson, A.J., Jakeman, L.B., McTigue, D.M., and Popovich, P.G. (2006). Basso Mouse Scale for locomotion detects differences in recovery after spinal cord injury in five common mouse strains. J. Neurotrauma 23, 635-659.

Bindels, D.S., Haarbosch, L., Van Weeren, L., Postma, M., Wiese, K.E., Mastop, M., Aumonier, S., Gotthard, G., Royant, A., Hink, M.A., et al. (2016). MScarlet: A bright monomeric red fluorescent protein for cellular imaging. Nat. Methods 14, 53-56.

Van Bockstaele, E.J., Pieribone, V.A., and Aston-Jones, G. (1989). Diverse afferents converge on the nucleus paragigantocellularis in the rat ventrolateral medulla: Retrograde and anterograde tracing studies. J. Comp. Neurol. 290, 561-584.

Brownstone, R.M., and Chopek, J.W. (2018). Reticulospinal systems for tuning motor commands. Front. Neural Circuits 12, 1-10.

Caggiano, V., Leiras, R., Goñi-Erro, H., Masini, D., Bellardita, C., Bouvier, J., Caldeira, V., Fisone, G., and Kiehn, O. (2018). Midbrain circuits that set locomotor speed and gait selection. Nature 553, 455-460.

Campbell, B.C., Nabel, E.M., Murdock, M.H., Lao-Peregrin, C., Tsoulfas, P., Blackmore, M.G., Lee, F.S., Liston, C., Morishita, H., and Petsko, G.A. (2020). mGreenLantern: a bright monomeric fluorescent protein with rapid expression and cell filling properties for neuronal imaging. Proc. Natl. Acad. Sci. U. S. A. 117, 30710-30721.

Capelli, P., Pivetta, C., Esposito, M.S., and Arber, S. (2017). Locomotor speed control circuits in the caudal brainstem. Nature 551, 373-377.

Chakraborty, T., Driscoll, M.K., Jeffery, E., Murphy, M.M., Roudot, P., Chang, B.J., Vora, S., Wong, W.M., Nielson, C.D., Zhang, H., et al. (2019). Light-sheet microscopy of cleared tissues with isotropic, subcellular resolution. Nat. Methods 16, 1109-1113.

Chen, T.W., Wardill, T.J., Sun, Y., Pulver, S.R., Renninger, S.L., Baohan, A., Schreiter, E.R., Kerr, R.A., Orger, M.B., Jayaraman, V., et al. (2013). Ultrasensitive fluorescent proteins for imaging neuronal activity. Nature 499, 295-300.

Cho, Y., Shi, R., and Borgens, R.B. (2010). Chitosan produces potent neuroprotection and physiological recovery following traumatic spinal cord injury. J. Exp. Biol. 213, 1513-1520.

Claudi, F., Tyson, A.L., Petrucco, L., Margrie, T.W., Portugues, R., and Branco, T. (2021). Visualizing anatomically registered data with brainrender. Elife 10.

Cornwall, J., Cooper, J.D., and Phillipson, O.T. (1990). Afferent and efferent connections of the laterodorsal tegmental nucleus in the rat. Brain Res. Bull. 25, 271-284. 
Davidsson, M., Wang, G., Aldrin-Kirk, P., Cardoso, T., Nolbrant, S., Hartnor, M., Mudannayake, J., Parmar, M., and Björklund, T. (2019). A systematic capsid evolution approach performed in vivo for the design of AAV vectors with tailored properties and tropism. Proc. Natl. Acad. Sci. U. S. A. 116, 27053-27062.

ten Donkelaar, H.J. (2000). Development and Regenerative Capacity of Descending Supraspinal Pathways in Tetrapods: A Comparative Approach (Berlin, Heidelberg: Springer Berlin Heidelberg).

Economo, M.N., Clack, N.G., Lavis, L.D., Gerfen, C.R., Svoboda, K., Myers, E.W., and Chandrashekar, J. (2016). A platform for brain-wide imaging and reconstruction of individual neurons. Elife 5, e10566.

Esposito, M.S., Capelli, P., and Arber, S. (2014). Brainstem nucleus MdV mediates skilled forelimb motor tasks. Nature 508, 351-356.

Fink, K.L., and Cafferty, W.B.J. (2016). Reorganization of Intact Descending Motor Circuits to Replace Lost Connections After Injury.

Flumerfelt, B.A., and Gwyn, D.G. (1974). Proceedings: The red nucleus of the rat: its organization and interconnexions. J. Anat. 118, 374, 376.

Fouad, K., Popovich, P.G., Kopp, M.A., and Schwab, J.M. (2021). The neuroanatomicalfunctional paradox in spinal cord injury. Nat. Rev. Neurol. 17, 53-62.

Frezel, N., Platonova, E., Voigt, F.F., Mateos, J.M., Kastli, R., Ziegler, U., Karayannis, T., Helmchen, F., Wildner, H., and Zeilhofer, H.U. (2020). In-Depth Characterization of Layer 5 Output Neurons of the Primary Somatosensory Cortex Innervating the Mouse Dorsal Spinal Cord. Cereb. Cortex Commun. 1, 1-16.

Glees, P. (1946). Terminal degeneration within the central nervous system as studied by a new silver method. J. Neuropathol. Exp. Neurol. 5, 54-59.

Gong, H., Zeng, S., Yan, C., Lv, X., Yang, Z., Xu, T., Feng, Z., Ding, W., Qi, X., Li, A., et al. (2013). Continuously tracing brain-wide long-distance axonal projections in mice at a onemicron voxel resolution. Neuroimage 74, 87-98.

de Groat, W.C., Griffiths, D., and Yoshimura, N. (2015). Neural control of the lower urinary tract. Compr. Physiol. 5, 327-396.

Hermann, G.E., Holmes, G.M., Rogers, R.C., Beattie, M.S., and Bresnahan, J.C. (2003).

Descending spinal projections from the rostral gigantocellular reticular nuclei complex. J. Comp. Neurol. 455, 210-221.

Hoff, E.C., and C H ff, B.E. (1932). The distribution of the spinal terminals (boutons) of the pyramidal tract, determined by experimental degeneration. Proc. R. Soc. London. Ser. B, Contain. Pap. a Biol. Character 111, 226-237.

Holstege, G. (2005). Central nervous system control of ejaculation. World J. Urol. 23, 109-114.

Kamiyama, T., Kameda, H., Murabe, N., Fukuda, S., Yoshioka, N., Mizukami, H., Ozawa, K., and Sakurai, M. (2015). Corticospinal tract development and spinal cord innervation differ 
between cervical and lumbar targets. J. Neurosci. 35, 1181-1191.

Kim, Y., Venkataraju, K.U., Pradhan, K., Mende, C., Taranda, J., Turaga, S.C., ArgandaCarreras, I., Ng, L., Hawrylycz, M.J., Rockland, K.S., et al. (2015). Mapping social behaviorinduced brain activation at cellular resolution in the mouse. Cell Rep. 10, 292-305.

Kozicz, T., Bittencourt, J.C., May, P.J., Reiner, A., Gamlin, P.D.R., Palkovits, M., Horn, A.K.E., Toledo, C.A.B., and Ryabinin, A.E. (2011). The Edinger-Westphal nucleus: a historical, structural, and functional perspective on a dichotomous terminology. J. Comp. Neurol. 519, 1413-1434.

Kuypers H., and Martin, G. (1982). Descending Pathways to the Spinal Cord. Prog. Brain Res. 57.

Kwon, B.K., Liu, J., Messerer, C., Kobayashi, N.R., McGraw, J., Oschipok, L., and Tetzlaff, W. (2002). Survival and regeneration of rubrospinal neurons 1 year after spinal cord injury. Proc. Natl. Acad. Sci. U. S. A. 99, 3246-3251.

Lakke, E.A. (1997). The projections to the spinal cord of the rat during development: a timetable of descent. Adv. Anat. Embryol. Cell Biol. 135.

Lemon, R.N. (2008). Descending Pathways in Motor Control. Annu. Rev. Neurosci. 31, 195218.

Leong, S.K., Shieh, J.Y., and Wong, W.C. (1984). Localizing spinal-cord-projecting neurons in adult albino rats. J. Comp. Neurol. 228, 1-17.

Liang, H., Paxinos, G., and Watson, C. (2011). Projections from the brain to the spinal cord in the mouse. Brain Struct. Funct. 215, 159-186.

Liang, H., Paxinos, G., and Watson, C. (2012). The red nucleus and the rubrospinal projection in the mouse. Brain Struct. Funct. 217, 221-232.

Liang, H., Watson, C., and Paxinos, G. (2016). Terminations of reticulospinal fibers originating from the gigantocellular reticular formation in the mouse spinal cord. Brain Struct. Funct. 221, 1623-1633.

Lu, P., Blesch, A., Graham, L., Wang, Y., Samara, R., Banos, K., Haringer, V., Havton, L., Weishaupt, N., Bennett, D., et al. (2012). Motor axonal regeneration after partial and complete spinal cord transection. J. Neurosci. 32, 8208-8218.

McCrea, R.A., and Horn, A.K.E. (2006). Nucleus prepositus. Prog. Brain Res. 151, 205-230.

Mtui, E.P., Anwar, M., Reis, D.J., and Ruggiero, D.A. (1995). Medullary visceral reflex circuits: Local afferents to nucleus tractus solitarii synthesize catecholamines and project to thoracic spinal cord. J. Comp. Neurol. 351, 5-26.

Niedworok, C.J., Brown, A.P.Y., Jorge Cardoso, M., Osten, P., Ourselin, S., Modat, M., and Margrie, T.W. (2016). AMAP is a validated pipeline for registration and segmentation of highresolution mouse brain data. Nat. Commun. 7, 1-9.

Nudo, R.J., and Masterton, R.B. (1988). Descending pathways to the spinal cord: A comparative study of 22 mammals. J. Comp. Neurol. 277, 53-79. 
Oh, S.W., Harris, J.A., Ng, L., Winslow, B., Cain, N., Mihalas, S., Wang, Q., Lau, C., Kuan, L., Henry, A.M., et al. (2014). A mesoscale connectome of the mouse brain. Nature 508, 207-214.

Peever, J., and Fuller, P.M. (2016). Neuroscience: A Distributed Neural Network Controls REM Sleep. Curr. Biol. 26, R34-R35.

Perreault, M.C., and Giorgi, A. (2019). Diversity of reticulospinal systems in mammals. Curr. Opin. Physiol. 8, 161-169.

Van Den Pol, A.N. (1999). Hypothalamic hypocretin (orexin): Robust innervation of the spinal cord. J. Neurosci. 19, 3171-3182.

Rodriguez, E.A., Campbell, R.E., Lin, J.Y., Lin, M.Z., Miyawaki, A., Palmer, A.E., Shu, X., Zhang, J., and Tsien, R.Y. (2017). The Growing and Glowing Toolbox of Fluorescent and Photoactive Proteins. Trends Biochem. Sci. 42, 111-129.

Roseberry, T.K., Lee, A.M., Lalive, A.L., Wilbrecht, L., Bonci, A., and Kreitzer, A.C. (2016). Cell-Type-Specific Control of Brainstem Locomotor Circuits by Basal Ganglia. Cell 164, 526537.

Ruder, L., Schina, R., Kanodia, H., Valencia-Garcia, S., Pivetta, C., and Arber, S. (2021). A functional map for diverse forelimb actions within brainstem circuitry. Nature 590, 445-450.

Sluka, K.A., and Westlund, K.N. (1992). Spinal projections of the locus coeruleus and the nucleus subcoeruleus in the Harlan and the Sasco Sprague-Dawley rat. Brain Res. 579, 67-73.

Soderblom, C., Lee, D.-H., Dawood, A., Carballosa, M., Santamaria, A.J., Benavides, F.D., Jergova, S., Grumbles, R.M., Thomas, C.K., Park, K.K., et al. (2015). 3D Imaging of Axons in Transparent Spinal Cords from Rodents and Nonhuman Primates. ENeuro 2, ENEURO.000115.2015.

Steward, O., Popovich, P.G., Dietrich, W.D., and Kleitman, N. (2012). Replication and reproducibility in spinal cord injury research. Exp. Neurol. 233, 597-605.

Steward, O., Yee, K.M., Metcalfe, M., Willenberg, R., Luo, J., Azevedo, R., Martin-Thompson, J.H., and Gandhi, S.P. (2021). Rostro-Caudal Specificity of Corticospinal Tract Projections in Mice. Cereb. Cortex 31, 2322-2344.

Strack, R. (2021). Single-objective light sheet microscopy. Nat. Methods 18, 28.

Swanson, L.W., and Kuypers, H.G.J.M. (1980). The paraventricular nucleus of the hypothalamus: Cytoarchitectonic subdivisions and organization of projections to the pituitary, dorsal vagal complex, and spinal cord as demonstrated by retrograde fluorescence doublelabeling methods. J. Comp. Neurol. 194, 555-570.

Takakusaki, K., Chiba, R., Nozu, T., and Okumura, T. (2016). Brainstem control of locomotion and muscle tone with special reference to the role of the mesopontine tegmentum and medullary reticulospinal systems. J. Neural Transm. 123, 695-729.

Tennant, K.A., Adkins, D.L., Donlan, N.A., Asay, A.L., Thomas, N., Kleim, J.A., and Jones, T.A. (2011). The organization of the forelimb representation of the C57BL/6 mouse motor cortex as defined by intracortical microstimulation and cytoarchitecture. Cereb. Cortex 21, 865-876. 
Tervo, D.G.R., Hwang, B.-Y., Viswanathan, S., Gaj, T., Lavzin, M., Ritola, K.D., Lindo, S., Michael, S., Kuleshova, E., Ojala, D., et al. (2016). A Designer AAV Variant Permits Efficient Retrograde Access to Projection Neurons. Neuron 92, 372-382.

Tuszynski, M.H., and Steward, O. (2012). Concepts and Methods for the Study of Axonal Regeneration in the CNS. Neuron 74, 777-791.

Tyson, A.L., and Margrie, T.W. (2021). Mesoscale microscopy for micromammals: image analysis tools for understanding the rodent brain.

Tyson, A.L., Rousseau, C. V., Niedworok, C.J., Keshavarzi, S., Tsitoura, C., and Margrie, T.W. (2020). A deep learning algorithm for 3D cell detection in whole mouse brain image datasets. BioRxiv 2020.10.21.348771.

Ueda, H.R., Ertürk, A., Chung, K., Gradinaru, V., Chédotal, A., Tomancak, P., and Keller, P.J. (2020). Tissue clearing and its applications in neuroscience. Nat. Rev. Neurosci. 21, 61-79.

Verstegen, A.M.J., Vanderhorst, V., Gray, P.A., Zeidel, M.L., and Geerling, J.C. (2017). Barrington's nucleus: Neuroanatomic landscape of the mouse "pontine micturition center." J. Comp. Neurol. 525, 2287-2309.

Wang, Z., Maunze, B., Wang, Y., Tsoulfas, P., and Blackmore, M.G. (2018). Global connectivity and function of descending spinal input revealed by 3D microscopy and retrograde transduction. J. Neurosci. 1196-18.

Xu, X.M., Chen, A., Guénard, V., Kleitman, N., and Bunge, M.B. (1997). Bridging Schwann cell transplants promote axonal regeneration from both the rostral and caudal stumps of transected adult rat spinal cord. J. Neurocytol. 26, 1-16.

Yu, M., and Wang, S.-M. (2020). Neuroanatomy, Interstitial Nucleus of Cajal (StatPearls Publishing).

Zholudeva, L. V., Abraira, V.E., Satkunendrarajah, K., McDevitt, T.C., Goulding, M.D., Magnuson, D.S.K., and Lane, M.A. (2021). Spinal interneurons as gatekeepers to neuroplasticity after injury or disease. In Journal of Neuroscience, (Society for Neuroscience), pp. 845-854.

Zhou, J.J., Ma, H.J., Shao, J.Y., Pan, H.L., and Li, D.P. (2019). Impaired Hypothalamic Regulation of Sympathetic Outflow in Primary Hypertension. Neurosci. Bull. 35, 124-132. 